ACCEPted for PUblication in ApJ

Preprint typeset using $\mathrm{LAT}_{\mathrm{E} X} \mathrm{X}$ style emulateapj v. 5/2/11

\title{
INTERNAL KINEMATICS OF GROUPS OF GALAXIES IN THE SLOAN DIGITAL SKY SURVEY DATA RELEASE 7
}

\author{
Cheng Li ${ }^{1}$, Y. P. Jing ${ }^{1}$, Shude MaO ${ }^{2,3}$, Jiaxin Han ${ }^{1}$, Qiuying Peng ${ }^{1}$, Xiaohu Yang ${ }^{1}$, \\ H. J. Mo ${ }^{4}$, Frank VAN DEN Bosch ${ }^{5}$ \\ Accepted for publication in ApJ
}

\begin{abstract}
We present measurements of the velocity dispersion profile (VDP) for galaxy groups in the final data release of the Sloan Digital Sky Survey (SDSS). For groups of given mass we estimate the redshift-space cross-correlation function (CCF) with respect to a reference galaxy sample, $\xi^{(s)}\left(r_{p}, \pi\right)$, the projected $\mathrm{CCF}, w_{p}\left(r_{p}\right)$, and the real-space $\mathrm{CCF}, \xi_{c g}(r)$. The VDP is then extracted from the redshift distortion in $\xi^{(s)}\left(r_{p}, \pi\right)$, by comparing $\xi^{(s)}\left(r_{p}, \pi\right)$ with $\xi_{c g}(r)$. We find that the velocity dispersion (VD) within virial radius $\left(R_{200}\right)$ shows a roughly flat profile, with a slight increase at radii below $\sim 0.3 R_{200}$ for high mass systems. The average VD within the virial radius, $\sigma_{v}$, is a strongly increasing function of central galaxy mass. We apply the same methodology to $N$-body simulations with the concordance $\Lambda$ cold dark matter cosmology but different values of the density fluctuation parameter $\sigma_{8}$, and we compare the results to the SDSS results. We show that the $\sigma_{v}-M_{*}$ relation from the data provides stringent constraints on both $\sigma_{8}$ and $\sigma_{m s}$, the dispersion in $\log M_{*}$ of central galaxies at fixed halo mass. Our best-fitting model suggests $\sigma_{8}=0.86 \pm 0.03$ and $\sigma_{m s}=0.16 \pm 0.03$. The slightly higher value of $\sigma_{8}$ compared to the WMAP7 result might be due to a smaller matter density parameter assumed in our simulations. Our VD measurements also provide a direct measure of the dark matter halo mass for central galaxies of different luminosities and masses, in good agreement with the results obtained by Mandelbaum et al. (2006) from stacking the gravitational lensing signals of the SDSS galaxies.

Subject headings: dark matter - galaxies: halos - large-scale structure - method: statistical
\end{abstract}

\section{INTRODUCTION}

Satellite galaxies are an important tracer of the potential well within which they reside, as they can be observed to much larger radii than other tracers. The potential well is determined by both the dark matter particles and baryons. In the past one and a half decade, $N$-body simulations provided excellent understanding of the mass profiles and shapes of dark matter haloes. For example, most haloes are well-fitted by the Navarro et al. (1997, hereafter NFW) profile, while their shapes are triaxial ellipsoids (Jing \& Suto 2002). However, it is expected that baryonic condensation at the centers of dark matter haloes may modify the inner profiles significantly, particularly on the galaxy scale (White \& Rees 1978; Blumenthal et al. 1986). Satellite galaxies provide a valuable probe of the dark matter halo profiles and the baryonic condensation processes.

On galaxy scale, satellites in the Milky Way provides the best dynamical constraint on its mass profile out to about $200 \mathrm{kpc}$ (Kochanek 1996; Wilkinson \& Evans 1999). Stellar-dynamical analysis of massive early-type

\footnotetext{
leech@shao.ac.cn

${ }^{1}$ Partner Group of the Max Planck Institute for Astrophysics at the Shanghai Astronomical Observatory and Key Laboratory for Research in Galaxies and Cosmology of Chinese Academy of Sciences, Nandan Road 80, Shanghai 200030, China

2 National Astronomical Observatories, Chinese Academy of Sciences, Beijing 100012, China

3 Jodrell Bank Centre for Astrophysics, University of Manchester, Alan Turing Building, Manchester M13 9PL, UK

${ }^{4}$ Department of Astronomy, University of Massachusetts, Amherst MA 01003-9305, USA

${ }^{5}$ Astronomy Department, Yale University, PO Box 208101, New Haven, CT 06520-8101, USA
}

galaxies also provide the kinematics of the inner few kpc, which seems to show the density profile is roughly isothermal (Koopmans et al. 2006). This is much steeper than the inner slope expected from the NFW profile, which suggests that baryonic processes have modified the inner profiles, consistent with theoretical expectations (e.g. Gnedin et al. 2004; Lin et al. 2006). For clusters of galaxies, using the CNOC1 survey Carlberg et al. (1997b) showed that for their clusters, the line of sight velocity rises from 0.1 virial radius, reaches a peak around 0.3 virial radius and then shows a roughly flat profile with a very slight decline. The line of sight velocity dispersion profile is consistent with that derived by Łokas \& Mamon (2003) from a detailed study of the nearby Coma cluster. The CNOC1 data have been used by van der Marel et al. (2000) to derive the mass distribution of clusters.

There have also been many studies that measure the average line-of-sight (los) velocity dispersion of the satellite galaxies within groups or clusters in order to infer the mass of their host dark matter halo. Early studies were mostly limited to rich galaxy clusters with a large number of satellite galaxies (e.g. Carlberg et al. 1996, 1997a). For less massive systems, halo mass estimates are usually obtained for central galaxies of similar luminosity or stellar mass by stacking the kinematics of their satellite galaxies (e.g. Erickson et al. 1987; Zaritsky et al. 1993; Zaritsky \& White 1994; Zaritsky et al. 1997). Taking advantage of the large redshift surveys, in particular the Two-degree Field Galaxy Redshift Survey (2dFGRS; Colless et al. 2001) and the Sloan Digital Sky Survey (SDSS; York et al. 2000), recent studies have applied this technique to large samples of satellite galaxies and studied the dependence of the los velocity dispersion on 
galaxy properties such as luminosity, stellar mass and optical color (e.g. McKay et al. 2002; Brainerd \& Specian 2003; Prada et al. 2003; van den Bosch et al. 2004; Becker et al. 2007; Conroy et al. 2007b; Norberg et al. 2008; More et al. 2009b, 2011). These studies have well established that the los velocity dispersion increases with galaxy luminosity and mass, consistent with the theoretical expectation that more massive galaxies are hosted by more massive halos. In addition, More et al. (2009b) found that the luminosity-halo mass relation may differ with color, while the stellar mass-halo mass relation is less color-dependent.

In this work we measure the velocity dispersion profile of satellite galaxies in groups by modelling the redshift distortions in the two-point cross-correlation function between galaxies and groups of galaxies. It has been well established that, the two-point autocorrelation function (2PCF) of galaxies measured from redshift surveys is distorted along the line of sight due to the peculiar motions of galaxies. On small scales the $2 \mathrm{PCF}$ is stretched along the line of sight, called 'Finger-of-God' (FoG) effect, and on large scales it is squashed due to the global infall of galaxies towards high-density regions (Kaiser 1987). Thus the redshift distortion (RSD) in $2 \mathrm{PCF}$ contains useful information about the relative motions of galaxies, and has been used to measure the so-called pairwise velocity dispersion (PVD) of different classes of galaxies by many authors (Davis \& Peebles 1983; Mo et al. 1993; Fisher et al. 1994; Zurek et al. 1994; Marzke et al. 1995; Somerville et al. 1997; Jing et al. 1998; Zehavi et al. 2002; Hawkins et al. 2003; Jing \& Börner 2004; Li et al. 2006a). These measurements have been used to test/constrain both semi-analytic models of galaxy formation (e.g. Li et al. 2007) and halo occupation distribution models of galaxy distribution (e.g. Jing \& Börner 2004; Slosar et al. 2006; Tinker et al. 2007; van den Bosch et al. 2007), as well as cosmological parameters (e.g. Jing et al. 1998; Yang et al. 2004; Wang et al. 2008; Cabré \& Gaztañaga 2009).

Here we use the same methodology as used in these previous studies, except that we use the galaxy-group crosscorrelation function to probe the velocity distributions of galaxies with respect to dark matter haloes. When compared to the aforementioned 'direct' measurements, our method has the advantage that it is not influenced by the so-called 'interlopers', i.e. mis-identified group members (see Yang et al. 2005a, for detailed discussion). The cross-correlation functions between groups and galaxies have been determined by Yang et al. (2005b) based on an earlier data release of the Sloan Digital Sky Survey (SDSS; York et al. 2000). In this study we make use of a sample of about 16,000 groups which is constructed from the final data release (DR7; Abazajian et al. 2009) of the SDSS using the halo-based group finding algorithm of Yang et al. (2007). We estimate both the redshift-space cross-correlation function $\xi^{(s)}\left(r_{p}, \pi\right)$ and the real-space cross-correlation function $\xi_{c g}(r)$, between a given subsample of groups and a reference sample of galaxies selected from the SDSS. The velocity dispersion profile for each subsample is then derived by modelling the redshift distortion in $\xi^{(s)}\left(r_{p}, \pi\right)$. As we will show, the velocity dispersion profiles within the virial radius can be reliably determined using the current data over a wide range in central galaxy luminosity and mass. More interestingly, comparisons with a set of high-resolution $N$-body simulations show that the average velocity dispersion within the virial radius as a function of central galaxy mass provides stringent constraints on both the density fluctuation parameter $\sigma_{8}$, and the correlation of the stellar mass of galaxies with the dark matter mass of their host halos.

Throughout this paper we assume a cosmology model with the density parameter $\Omega_{m}=0.27$ and the cosmological constant $\Omega_{\Lambda}=0.73$, and a Hubble constant $H_{0}=100 h \mathrm{kms}^{-1} \mathrm{Mpc}^{-1}$ with $h=0.7$.

\section{DATA}

\subsection{Group catalog}

The galaxy group catalog used in this paper is constructed by Yang et al. (2007) from sample dr72 of the New York University Value-Added Galaxy Catalogue (NYU-VAGC). The NYU-VAGC is a catalogue of local galaxies (mostly below $z \approx 0.3$ ) selected from the SDSS data release 7 (DR7; Abazajian et al. 2009), publicly available at http://sdss.physics.nyu.edu/vagc/, and is described in detail in Blanton et al. (2005). The main virtue of the NYU-VAGC is that it provides a detailed account of the selection effects in the survey, thus suitable for statistical studies of the galaxy distribution and large-scale structure in the local Universe.

To select the groups of galaxies, a modified version of the halo-based group-finding algorithm developed in Yang et al. (2005a) is applied to a sample of $\sim 6.4 \times 10^{5}$ galaxies selected from the NYU-VAGC sample dr72 with redshifts in the range $0.01 \leq z \leq 0.20$ and with a redshift completeness above $70 \%$. The reader is referred to Yang et al. (2005a) and Yang et al. (2007) for detailed description of the group finder. The group catalogue contains about half a million systems, of which the majority have only a single member. In this work we use a subset of $\sim 16,000$ groups that have at least three member galaxies.

We use the most massive galaxy member of each group as the group center which is called the "central" galaxy in what follows. The stellar mass of each central galaxy accompanies the NYU-VAGC release, which is estimated based on its redshift and the five-band magnitudes from SDSS photometric data, as described in detail in Blanton \& Roweis (2007). This estimate corrects implicitly for dust and assumes a universal stellar initial mass function (IMF) of Chabrier (2003) form. As demonstrated in Appendix A of Li \& White (2009), once all estimates are adapted to assume the same IMF, the Blanton \& Roweis masses agree quite well with those obtained from the simple, single-color estimator of Bell et al. (2003) and also with those derived by Kauffmann et al. (2003) from a combination of SDSS photometry and spectroscopy. In this work we use the "total masses" instead of the "Petrosian masses" used by Li \& White (2009), obtained by correcting the latter using SDSS "model magnitudes" (see Appendix A of Guo et al. 2010, for details).

From the group catalogue, we select 18 subsamples according to the stellar mass of the central galaxies, ranging from $\log \left(M_{*} / M_{\odot}\right)=10.0$ to 12.0. Each subsample includes central galaxies in a stellar mass interval 
TABLE 1

Subsamples OF GROUPS SELECTED ACCORDING TO THE STELlar MASS OF THEIR CENTRAL GALAXIES, INCLUDING THE STELLAR MASS RANGE, THE NUMBER OF GROUPS, THE MEAN STELLAR MASS AND THE MEAN LUMINOSITY OF EACH SUBSAMPLE, AS WELL AS THE ONE-DIMENSIONAL VELOCITY DISPERSION AND HALO MASS DETERMINED FOR EACH SUBSAMPLE.

\begin{tabular}{|c|c|c|c|c|c|}
\hline $\begin{array}{c}\log \left(M_{*} / M_{\odot}\right) \\
(1)\end{array}$ & $\begin{array}{l}N_{\text {group }} \\
\text { (2) }\end{array}$ & $\begin{array}{c}\log \left(\left\langle M_{*} / M_{\odot}\right\rangle\right) \\
(3)\end{array}$ & $\begin{array}{c}\log \left(\left\langle L / L_{\odot}\right\rangle\right) \\
(4)\end{array}$ & $\begin{array}{c}\sigma_{v} / k m s^{-1} \\
(5)\end{array}$ & $\begin{array}{c}\log \left(M_{h} / M_{\odot}\right) \\
(6)\end{array}$ \\
\hline$[10.3,10.6)$ & 913 & 10.49 & 10.33 & $133 \pm 11$ & $12.17 \pm 0.13$ \\
\hline$[10.4,10.7)$ & 1415 & 10.59 & 10.40 & $139 \pm 8$ & $12.24 \pm 0.09$ \\
\hline$[10.5,10.8)$ & 2190 & 10.69 & 10.47 & $151 \pm 6$ & $12.36 \pm 0.06$ \\
\hline$[10.6,10.9)$ & 3145 & 10.78 & 10.53 & $166 \pm 5$ & $12.50 \pm 0.05$ \\
\hline$[10.7,11.0)$ & 4245 & 10.88 & 10.61 & $188 \pm 6$ & $12.69 \pm 0.05$ \\
\hline$[10.8,11.1)$ & 5257 & 10.97 & 10.69 & $216 \pm 7$ & $12.88 \pm 0.04$ \\
\hline$[10.9,11.2)$ & 6214 & 11.07 & 10.77 & $243 \pm 6$ & $13.04 \pm 0.04$ \\
\hline$[11.0,11.3)$ & 6537 & 11.16 & 10.85 & $291 \pm 8$ & $13.29 \pm 0.03$ \\
\hline$[11.1,11.4)$ & 6014 & 11.24 & 10.93 & $338 \pm 9$ & $13.49 \pm 0.03$ \\
\hline$[11.2,11.5)$ & 4685 & 11.33 & 11.01 & $385 \pm 10$ & $13.65 \pm 0.03$ \\
\hline$[11.3,11.6)$ & 3115 & 11.42 & 11.09 & $439 \pm 15$ & $13.82 \pm 0.04$ \\
\hline$[11.4,11.7)$ & 1806 & 11.51 & 11.18 & $522 \pm 22$ & $14.04 \pm 0.05$ \\
\hline$[11.5,11.8)$ & 903 & 11.60 & 11.26 & $591 \pm 32$ & $14.20 \pm 0.07$ \\
\hline$[11.6,11.9)$ & 374 & 11.69 & 11.35 & $654 \pm 38$ & $14.32 \pm 0.07$ \\
\hline
\end{tabular}

of 0.3 dex, with successive subsamples overlapping by 0.2 dex. As we will show below, the velocity dispersion profile cannot be reliably determined for subsamples below $\log \left(M_{*} / M_{\odot}\right)=10.3$. We will also ignore the most massive subsample with $11.9 \leq \log \left(M_{*} / M_{\odot}\right)<12.0$ when measuring the velocity dispersion profile, because its redshift-space cross-correlation function is noisy due to the small sample size. The stellar mass range, the number of groups, and the mean stellar mass and luminosity of the rest 14 subsamples are listed in the first four columns in Table 1. The luminosity of each galaxy is computed from its $r$-band absolute magnitude and the absolute magnitude of the Sun is assumed to be 4.76 mag following Blanton et al. (2003).

\subsection{Reference galaxy sample and random sample}

We have constructed a magnitude-limited galaxy sample from the NYU-VAGC sample dr72, which will serve as our reference sample when measuring the groupgalaxy cross-correlation functions. This consists of about half a million galaxies with $r<17.6,-24<M_{0.1} r<-16$ and redshifts in the range $0.01<z<0.2$. Here, $r$ is the $r$-band Petrosian apparent magnitude, corrected for Galactic extinction, and $M_{0.1} r$ is the $r$-band Petrosian absolute magnitude, corrected for evolution and $K$-corrected to its value at $z=0.1$. These selection criteria, with the exception of the redshift range, are the same as in our previous papers where we studied the clustering of galaxy luminosity and stellar mass (e.g. Li et al. 2012).

To obtain reliable estimates of the cross-correlation functions, which will form the basis of our study, the cross pair counts between the groups and the reference galaxies must be compared with the cross pair counts between the same set of groups and a 'random sample'. The random sample is unclustered but fills the same region of the sky and has the same position- and redshiftdependent selection effects as the reference sample. We have constructed our random sample from the observed reference sample itself, as described in detail in $\mathrm{Li}$ et al. (2006b). For each real galaxy we generate 10 sky positions at random within the mask of sample dr72, and we assign to each of them the redshift of the real galaxy. The resulting random sample is valid for clustering analyses, provided that the survey area is large enough so that structures in the real sample are wiped out by randomizing in angle, and that the effective depth of the survey does not vary from region to region. Both are true to good accuracy for our sample, which covers $\gtrsim 6000$ $\mathrm{deg}^{2}$, is complete down to $r=17.6$ and is little affected by foreground dust over the entire survey region. Extensive tests show that random samples constructed in this way produce indistinguishable results from those using the traditional method ( $\mathrm{Li}$ et al. 2006b).

\subsection{Simulations and dark matter subhalo catalogs}

In this paper, we use three $N$-body simulations and dark matter halo catalogs constructed from them to study the connections of galaxy velocity dispersions with halo masses and cosmological parameters. The simulations use $1024^{3}$ particles to follow the dark-matter distribution in a cubic region with $300 h^{-1} \mathrm{Mpc}$ on a side, corresponding to a particle mass of $1.87 \times 10^{9} h^{-1} \mathrm{M}_{\odot}$. These are obtained using an upgraded version of the particleparticle-particle-mesh $\left(\mathrm{P}^{3} \mathrm{M}\right)$ code of Jing \& Suto (1998, $2002)$, which has incorporated the multiple-level $\mathrm{P}^{3} \mathrm{M}$ gravity solver for high-density regions (Jing \& Suto 2000; Jing et al. 2007). The cosmology model assumed is a spatially flat $\Lambda$ CDM model with the same parameters $\Omega_{m}=$ $0.268, \Omega_{\Lambda}=0.732, \Omega_{b}=0.045, H_{0}=71 \mathrm{kms}^{-1} \mathrm{Mpc}^{-1}$ and $n=1$, except that a different density fluctuation parameter is adopted, which is $\sigma_{8}=0.75,0.85$ and 0.95 , for the three simulations respectively. The simulations are described in detail in Jing et al. (2007). The cosmology for the simulations is nearly identical to the cosmology we assume in this study (mentioned in the introduction).

Dark matter halos are identified for each of the simulation outputs using the standard Friends-of-Friends (FoF) algorithm with the linking length set to be 0.2 times the mean particle separation (Davis et al. 1985). The Hierarchical Bound-Tracing (HBT) algorithm recently developed by Han et al. (2011) is then applied to split the FoF halos into disjoint, self-bound subhalos. The HBT algorithm finds and traces dark matter subhalos in sim- 
ulations based on their merger hierarchy. Thus, when compared to previously existing subhalo finders, this new algorithm has the unique advantage that it is able to well resolve the subhalos in high density environment, while keeping strict physical track of their merger history. Inside each FoF halo, the subhalos are divided into two classes which are treated differently during tracing: 'central' subhalo and 'satellite' subhalos. The central subhalo is defined as the most massive subhalo of an FoF halo, is able to grow through accreting mass within the host halo and normally contains most of its mass. All the subhalos in an FoF halo except the central one are called satellite subhalos.

We determine the position and velocity of each subhalo to be the center of mass and bulk velocity of its core, consisting of $25 \%$ of its bound particles with the lowest potential. For each central subhalo, we define the virial radius, $R_{200}$, as the radius within which the mean density of dark matter particles is equal to 200 times the critical value. The virial mass of the central subhalo is then defined as the mass within $R_{200}$ :

$$
M_{200}=200 \rho_{\text {crit }} \frac{4 \pi}{3} R_{200}^{3}=100 G^{-1} H^{2}(z) R_{200}^{3} .
$$

For a satellite subhalo, we use the $M_{200}$ at the epoch when it was last the central subhalo in its host halo. Hereafter, we refer to both central and satellite subhalos as 'halos', and we refer to $M_{200}$ defined in this way as the 'halo mass', if not specifically pointed out.

\section{RESULTS}

\subsection{Group-galaxy cross-correlation functions}

In this work we estimate the velocity dispersion of galaxies by modelling the redshift distortions in groupgalaxy cross-correlation functions. Thus, for each subsample of groups listed in Table 1, we begin by estimating the redshift-space cross-correlation function, $\xi^{(s)}\left(r_{p}, \pi\right)$, between the group central galaxies and the galaxies in the reference sample, using the commonly-used estimator

$$
\xi^{(s)}\left(r_{p}, \pi\right)=\frac{N_{R}}{N_{G}} \frac{C G\left(r_{p}, \pi\right)}{C R\left(r_{p}, \pi\right)}-1 .
$$

Here $r_{p}$ and $\pi$ are the separations perpendicular and parallel to the line of sight; $N_{G}$ and $N_{R}$ are the number of galaxies in the reference sample and in the random sample; $C G\left(r_{p}, \pi\right)$ and $C R\left(r_{p}, \pi\right)$ are the cross pair counts between the group subsample and the reference sample, and between the group subsample and the random sample respectively, both with perpendicular separations in the bins $\log r_{p} \pm \frac{1}{2} \Delta \log r_{p}$ and with radial separations in the bins $\pi \pm \frac{1}{2} \Delta \pi$. To reduce sampling noise, the random sample is constructed with a number of particles that is 10 times the number of galaxies in the reference sample, i.e., $N_{R}=10 \times N_{G}$.

An estimate of the projected cross-correlation function, $w_{p}\left(r_{p}\right)$, is then obtained by integrating $\xi^{(s)}\left(r_{p}, \pi\right)$ over the line-of-sight separations $\pi$. We take

$$
w_{p}\left(r_{p}\right)=\int_{-\pi_{\max }}^{\pi_{\max }} \xi^{(s)}\left(r_{p}, \pi\right) d \pi=\sum_{i} \xi^{(s)}\left(r_{p}, \pi_{i}\right) \Delta \pi_{i},
$$

where we choose $\pi_{\max }=40 h^{-1} \mathrm{Mpc}$ as the outer limit for the integration depth (in order to limit noise from distant uncorrelated regions) so that the summation for computing $w_{p}\left(r_{p}\right)$ runs from $\pi_{1}=-39.5 h^{-1} \mathrm{Mpc}$ to $\pi_{80}=39.5 h^{-1} \mathrm{Mpc}$, given that we use bins of width $\Delta \pi_{i}=1 h^{-1} \mathrm{Mpc}$. We note that we have tried with different outer limits for the integration depth and the resulting $w_{p}\left(r_{p}\right)$ changes little. We have corrected the effect of fiber collisions in the SDSS data using the method described in Li et al. (2006c).

In Fig. 1, we show the projected cross-correlation functions determined in this way for some of our group subsamples. The errors on the cross-correlation function measurements are estimated using the bootstrap resampling technique (Barrow et al. 1984). We generate 100 bootstrap samples from the group subsample and compute the cross-correlation functions for each sample using the weighting scheme (but not the approximate formula) given by Mo et al. (1992). The errors are then given by the $1 \sigma$ scatter of the measurements among these bootstrap samples.

In all the cases the projected cross-correlation function can well be separated into two parts: a steeper inner part at separations below around $1 h^{-1} \mathrm{Mpc}$ and a flat outer part at larger separations. In the language of the halo model (e.g. Cooray \& Sheth 2002), the inner part is dominated by the 'one-halo' term where the pair counts are mostly galaxy pairs in the same halo (centralsatellite pairs in the case of cross-correlation functions as in this study), and the outer part is dominated by the 'two-halo' term where galaxy pairs are mostly in separate halos (central-central plus central-satellite pairs between different groups). The separation where the transition between the one-halo and two-halo terms occurs increases monotonically from $\sim 200 h^{-1} \mathrm{kpc}$ for the lowest-mass systems in our sample with $M_{*} \sim 10^{10} M_{\odot}$, up to $\sim 1 h^{-1} \mathrm{Mpc}$ for the most massive systems with $M_{*} \sim 10^{12} M_{\odot}$. The one-halo term shows a systematic change in shape with the mass of central galaxies, with steeper slopes at lower masses and flatter slopes at higher masses. This implies that the distribution of satellite galaxies in low-mass halos is more concentrated than that in high-mass halos. Moreover, the amplitude of the twohalo term increases with increasing mass, reflecting the tight correlation between the stellar mass of the central galaxies and the mass of their host dark matter halos. In contrast, the slope of the two-halo term seems to vary little, reflecting the known fact that the bias in the galaxy distribution is related in a very simple way to the bias in the distribution of dark haloes. The features seen in the group-galaxy cross-correlation functions are all well consistent with what we found in Yang et al. (2005b), but with smaller error bars thanks to the improvement of the data.

In the plane-parallel approximation, the projected cross-correlation function $w_{p}\left(r_{p}\right)$ is expected to be directly related to the real-space cross-correlation function $\xi_{c g}(r)$ by an Abel transform,

$$
w_{p}\left(r_{p}\right)=\int_{-\infty}^{\infty} \xi_{c g}\left(\sqrt{r_{p}^{2}+y^{2}}\right) d y=2 \int_{r_{p}}^{\infty} \frac{r \xi_{c g}(r)}{\sqrt{r^{2}-r_{p}^{2}}} d r .
$$




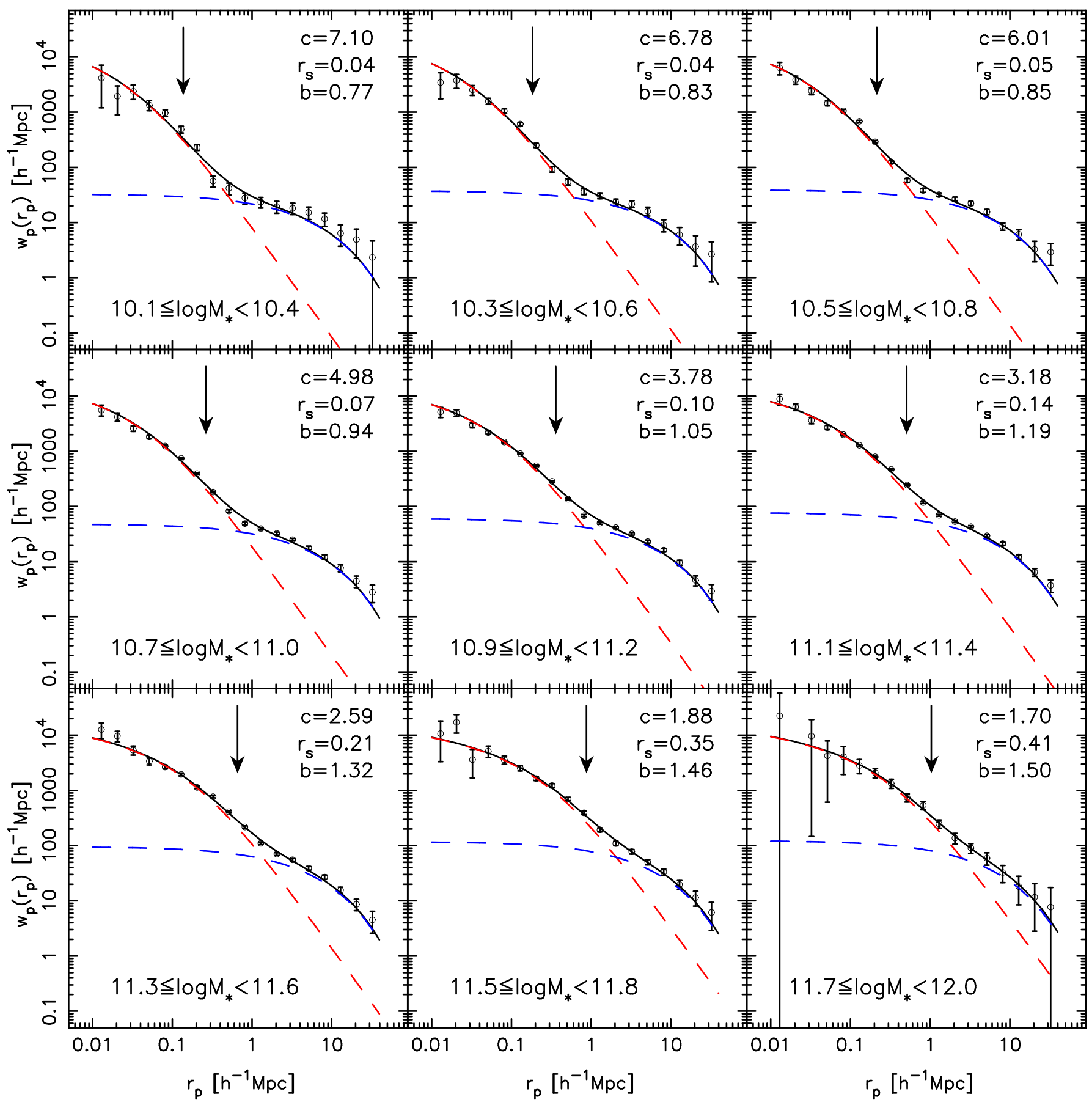

FIG. 1.- The projected cross-correlation function between the central galaxies of groups and the reference galaxies, estimated for some of the group subsamples listed in Table 1. The symbols with error bars are measured using the SDSS DR7 group catalogue. The solid lines are the best-fits of Eq.(4) to the data. The dashed and dotted lines are the small-scale component modelled by an NFW profile and the large-scale component that is a biased version of the projected linear two-point correlation function of dark matter in a WMAP7 cosmology. The stellar mass range of the group central galaxies and the best-fitting values of the model parameters are indicated in each panel (note that the parameter $r_{s}$ is in units of $h^{-1} \mathrm{Mpc}$ ). The virial radius $R_{200}$ given by the estimated halo masses listed in Table 1 is indicated by an vertical arrow in every panel. See the text for details. 
the $w_{p}\left(r_{p}\right)$ measurements as discussed above, as well as previous theoretical studies of the cross-correlation function between halo centers and dark matter particles (e.g. Hayashi \& White 2008), we model the real-space crosscorrelation function $\xi_{c g}(r)$ as a linear combination of two components as follows,

$$
\xi_{c g}(r)=\xi_{\mathrm{NFW}}(r)+b \xi_{d m}(r)
$$

The first component, $\xi_{\mathrm{NFW}}(r)$, is the NFW profile,

$$
\xi_{\mathrm{NFW}}(r)=\frac{\delta_{c}}{\left(r / r_{s}\right)\left(1+r / r_{s}\right)^{2}},
$$

where

$$
\delta_{c}=\frac{200}{3 \Omega_{m}} \frac{c^{3}}{\ln (1+c)-c /(1+c)} .
$$

The second component, $\xi_{d m}(r)$, is the linear two-point correlation function of dark matter at $z=0$ for the WMAP7 cosmology, obtained by Fourier transforming $P_{l}(k)$, the linear power spectrum calculated using the CAMB code of Lewis et al. (2000). Thus, the model has three free parameters: $c, r_{s}$ and $b$, and we will call them concentration parameter, characteristic radius and bias factor in what follows, though they may have different meanings from their original definitions in the literature.

For a given set of parameters, the model predicts both the real-space correlation function $\xi_{c g}(r)$ through Eqn. (5) and the projected function $w_{p}\left(r_{p}\right)$ through Eqn. (4). We determine a best-fitting model for each of our group subsamples by comparing the predicted $w_{p}\left(r_{p}\right)$ with the observed one, and we define the best-fitting model to be the one giving a minimum $\chi^{2}$. Our best models are plotted in Fig. 1 as solid black lines, and the two components of each model are plotted in red and blue dashed lines separately. The model parameters are also indicated. As can be seen, the two-component model provides a compact and accurate description of the observed $w_{p}\left(r_{p}\right)$ for all our group subsamples. It is interesting that all the model parameters show systematic trends when one goes from the lowest-mass groups to the highest-mass groups, in the sense that both $r_{s}$ and $b$ increase with mass, while the concentration parameter, $c$, decreases with mass. This is again consistent with the picture that more massive galaxies are hosted by more massive dark halos within which the galaxy distribution is less concentrated and extends to a larger radius.

Moreover, the concentration parameter of satellite distribution as inferred from our best-fitting models is smaller than the concentration of dark matter halos given by high-resolution simulations (e.g. Zhao et al. 2009), implying that the distribution of galaxies in dark halos is more extended than dark matter particles. This is consistent with some previous studies (e.g. Yang et al. 2005b; Chen et al. 2006; More et al. 2009a), though other studies produced opposite results, especially for luminous red galaxies (e.g. Masjedi et al. 2006; Watson et al. 2010, 2012; Tal et al. 2012). Nevertheless, we would like to emphasize that our model parameters should not be overinterpreted, although they exhibit reasonable values and interesting trends with galaxy mass that seem to be easily understood from our knowledge of galaxy-halo connections.

In Fig. 2 we plot again the measurements and the bestfits of the projected cross-correlation function, as func- tions of both $r_{p}$ (left panel) and $r_{p} / R_{200}$ (right panel). The systematic trends of $w_{p}\left(r_{p}\right)$ with central galaxy mass as seen from Fig. 1 are more clearly seen here. These include the increase in the amplitude of the two-halo term on large scales, the change in the shape of the one-halo term on small scales, and the shift in the transition scale between the two terms on intermediate scales.

\subsection{Velocity dispersion profiles}

In the previous subsection we have obtained the groupgalaxy cross-correlation functions, both in redshift space, $\xi^{(s)}\left(r_{p}, \pi\right)$ and in real space, $\xi_{c g}(r)$. In Fig. 3 we show the contours of $\xi^{(s)}\left(r_{p}, \pi\right)$ for some of our group subsamples. Both the 'Finger-of-God' (FoG) effect on small scales and the infall effect (Kaiser 1987) on large scales are clearly visible: on small scales $\xi^{(s)}\left(r_{p}, \pi\right)$ is stretched in the $\pi$ direction and on large scales the contours are squashed. The FoG effect is caused by the peculiar, virialized motions of galaxies within dark matter haloes, and is much more pronounced in the more massive haloes, reflecting their larger velocity dispersions. Here, we model these redshift-space distortions in detail, in order to infer the velocity field in and around dark haloes.

Our method relies on the fact that the peculiar motions of galaxies affect only their radial distances in redshift space. Thus the information for peculiar velocities along the line of sight can be recovered by modelling the redshift-space cross-correlation function $\xi^{(s)}\left(r_{p}, \pi\right)$ as a convolution of the real-space function $\xi_{c g}(r)$ with the distribution function of the peculiar velocity $f\left(v_{c s}\right)$ :

$$
\xi^{(s)}\left(r_{p}, \pi\right)=\int f\left(v_{c s}\right) \xi_{c g}\left(\sqrt{r_{p}^{2}+\left(\pi-v_{c s}\right)^{2}}\right) d v_{c s},
$$

where $v_{c s}$ is the peculiar velocity of satellite galaxies relative to the central galaxy. We adopt a Gaussian form for $f\left(v_{c s}\right)$ :

$$
f\left(v_{c s}\right)=\frac{1}{\sqrt{2 \pi} \sigma_{v}} \exp \left[\frac{-\left(v_{c s}-\overline{v_{c s}}\right)^{2}}{2 \sigma_{v}^{2}}\right],
$$

where $\overline{v_{c s}}$ is the mean and $\sigma_{v}$ is the dispersion of the one dimensional peculiar velocities. Note that we have assumed that the distributions of the peculiar velocity and the velocity dispersion are isotropic. Assuming an infall model for $\overline{v_{c s}}(r)$, the velocity dispersion $\sigma_{v}$ can then be estimated as a function of the projected separation $r_{p}$ by comparing the observed $\xi^{(s)}\left(r_{p}, \pi\right)$ with the modelled one.

Following Croft et al. (1999), we use the non-linear spherical collapse model as our infall model,

$$
\overline{v_{c s}}(r)=v_{l}(r)[1+\delta(r)]^{-0.25} \exp \left[-\frac{\delta(r)}{\delta_{c u t}}\right] .
$$

The first factor at the right-hand side, $v_{l}$, is the infall velocity in the linear perturbation case, which depends on the distance to the local density maximum and is directly related to the density contrast (Peebles 1980):

$$
v_{l}(r)=-\frac{1}{3} H_{0} \Omega_{m}^{0.6} r \delta(r),
$$

where $\delta(r)$ is the overdensity inside radius $r$,

$$
\delta(r)=\frac{3 J_{3}^{c \rho}(r)}{r^{3}}, \quad J_{3}^{c \rho}(r)=\int_{0}^{r} \xi_{c \rho}(x) x^{2} d x .
$$



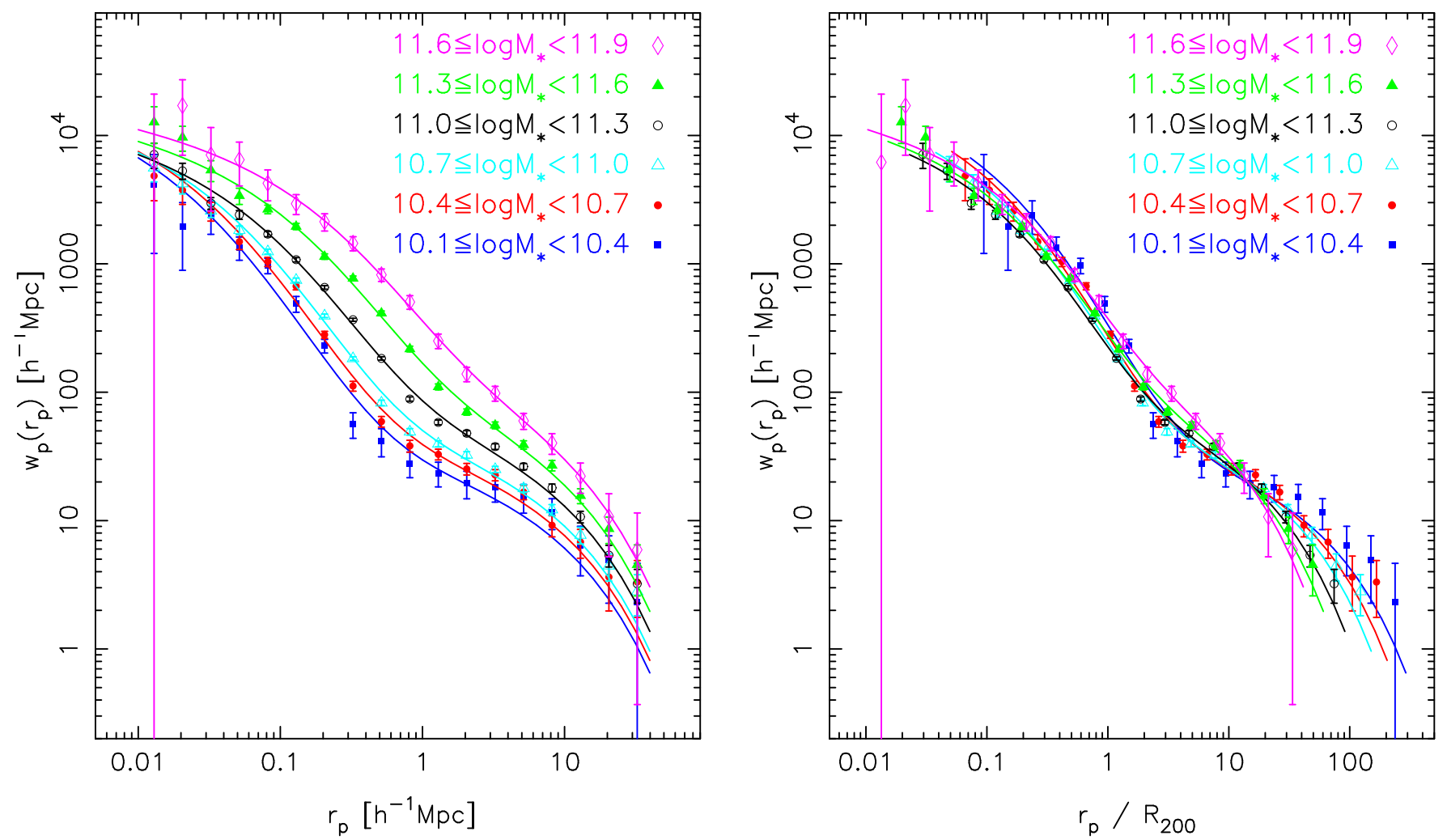

FIG. 2.- Left: the $w_{p}\left(r_{p}\right)$ measurements from the SDSS/DR7 group catalogue are plotted in symbols with error bars for some of our group subsamples, and are compared to the best-fits of Eqn.(4) to the data which are plotted in solid lines. Right: same as in the left panel except that the projected separation $r_{p}$ is scaled by the virial radius $R_{200}$ on the x-axis. The stellar mass ranges of the central galaxies are indicated.

Here $\xi_{c \rho}$ is the linear cross-correlation between galaxy clusters (or groups in our case) and matter. We use the simple linear biasing picture, so that

$$
J_{3}^{c \rho}(r)=\frac{J_{3}^{c g}(r)}{b}=\frac{1}{b} \int_{0}^{r} \xi_{c g}(x) x^{2} d x,
$$

where $\xi_{c g}$ is the group-galaxy cross-correlation function in real-space and $b$ is the linear bias factor in Eqn.(5), both of which have been obtained in the previous subsection for each of our group subsamples. The powerlaw part at the right-hand side in Eqn.(10) is introduced by Yahil (1985) (also used by Lilje \& Efstathiou 1989) as a good approximation to the exact solution for the non-linear collapse of spherically symmetric clusters. The exponential truncation in Eqn.(10) is suggested by Croft et al. (1999) in order to take into account the highly non-linear behavior of the infall velocity at high overdensities. Tests against N-body simulations showed that Eqn.(10) with $\delta_{\text {cut }}=50$ provides an accurate description of the average infall velocity of matter into galaxy clusters (Croft et al. 1999).

In Fig. 3 we plot the contours of our best-fitting models of $\xi^{(s)}\left(r_{p}, \pi\right)$ obtained by applying Eqn.(8) to the data. In Fig. 4 we plot the velocity dispersion, $\sigma_{v}$, obtained by applying Eqn.(8) to the data, as functions of both the projected separation $\left(r_{p}\right.$, left panel) and the separation scaled by the virial radius $\left(r_{p} / R_{200}\right.$, right panel). There are several interesting trends/features that can be read from this figure. First, at fixed radii the velocity dispersion is a strongly increasing function of central galaxy mass (thus halo mass), and the mass dependence becomes stronger for more massive systems. The velocity dispersion within the scales of the one-halo term ranges from $\sim 100 \mathrm{kms}^{-1}$ for the lowest-mass systems in our sample $\left(M_{*} \sim 2 \times 10^{10} M_{\odot}\right)$, up to $\sim 700 \mathrm{kms}^{-1}$ for the most massive systems with $M_{*} \sim 5 \times 10^{11} M_{\odot}$. Second, the velocity dispersion shows a roughly flat profile at radii smaller than a few $100 h^{-1} \mathrm{kpc}$, with a slight increase at the smallest radii for the massive systems with $M_{*} \gtrsim 3 \times 10^{11} M_{\odot}$. This echoes the early results revealed from CNOC1 survey by Carlberg et al. (1997b) who found that for their clusters the line-of-sight velocity showed a flat profile at radii larger than 0.3 virial radius with a very slight decline (see also Carlberg et al. 1996; Biviano \& Katgert 2004), as well as later studies on galaxy groups by Carlberg et al. (2001) who found flat or slowly rising profiles for small groups. Finally, the velocity dispersion profile exhibits a broad bump at around $1 \mathrm{Mpc}$, which is more pronounced for less massive systems. As we will show below, this is likely caused by contamination from neighboring larger halos where the high-speed galaxies contribute significantly to the velocity dispersion measurement of the smaller system.

\subsection{Understanding the origin of the bump at around 1 $M p c$}

In order to understand the origin of the bump at around $1 \mathrm{Mpc}$ in the velocity dispersion profile of the low-mass systems, we have performed the same analyses using our simulations and compared the results with the 
Li et al.
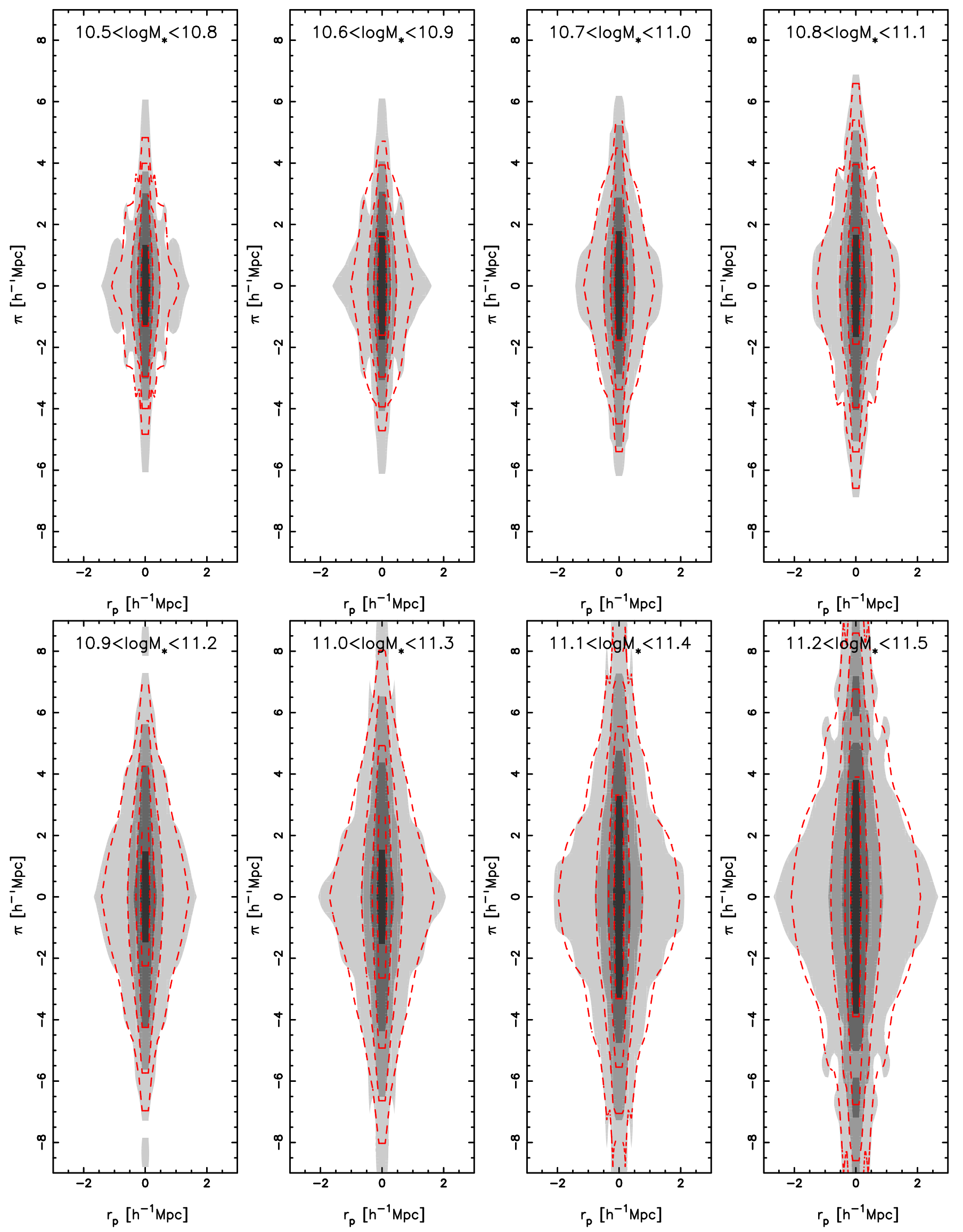

FIG. 3.- The redshift-space group-galaxy cross-correlation function $\xi^{(s)}\left(r_{p}, \pi\right)$ for groups with central galaxies in different stellar mass intervals, as indicated in each panel. The gray shaded regions show the measurements from the SDSS/DR7 data and the dashed red lines are the best-fits of Eqn.(8) to the data. 

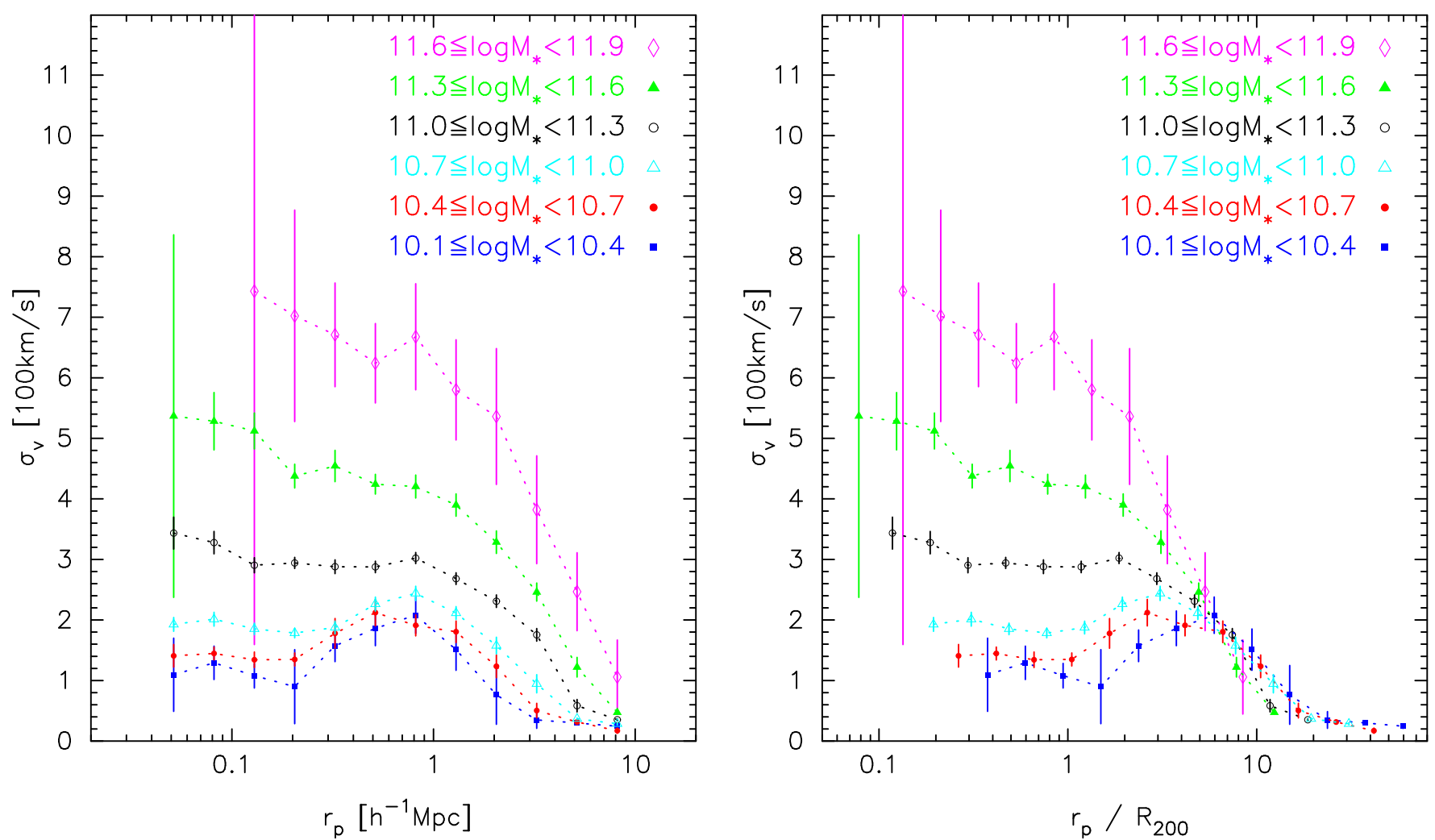

FIG. 4.- Left: the velocity dispersion profile for some of our group subsamples, obtained by applying Eqn.(8) to the data. Right: same as the left panel except that the projected separation $r_{p}$ is scaled by the virial radius $R_{200}$ on the x-axis. The stellar mass ranges of the group subsamples are indicated in both panels.
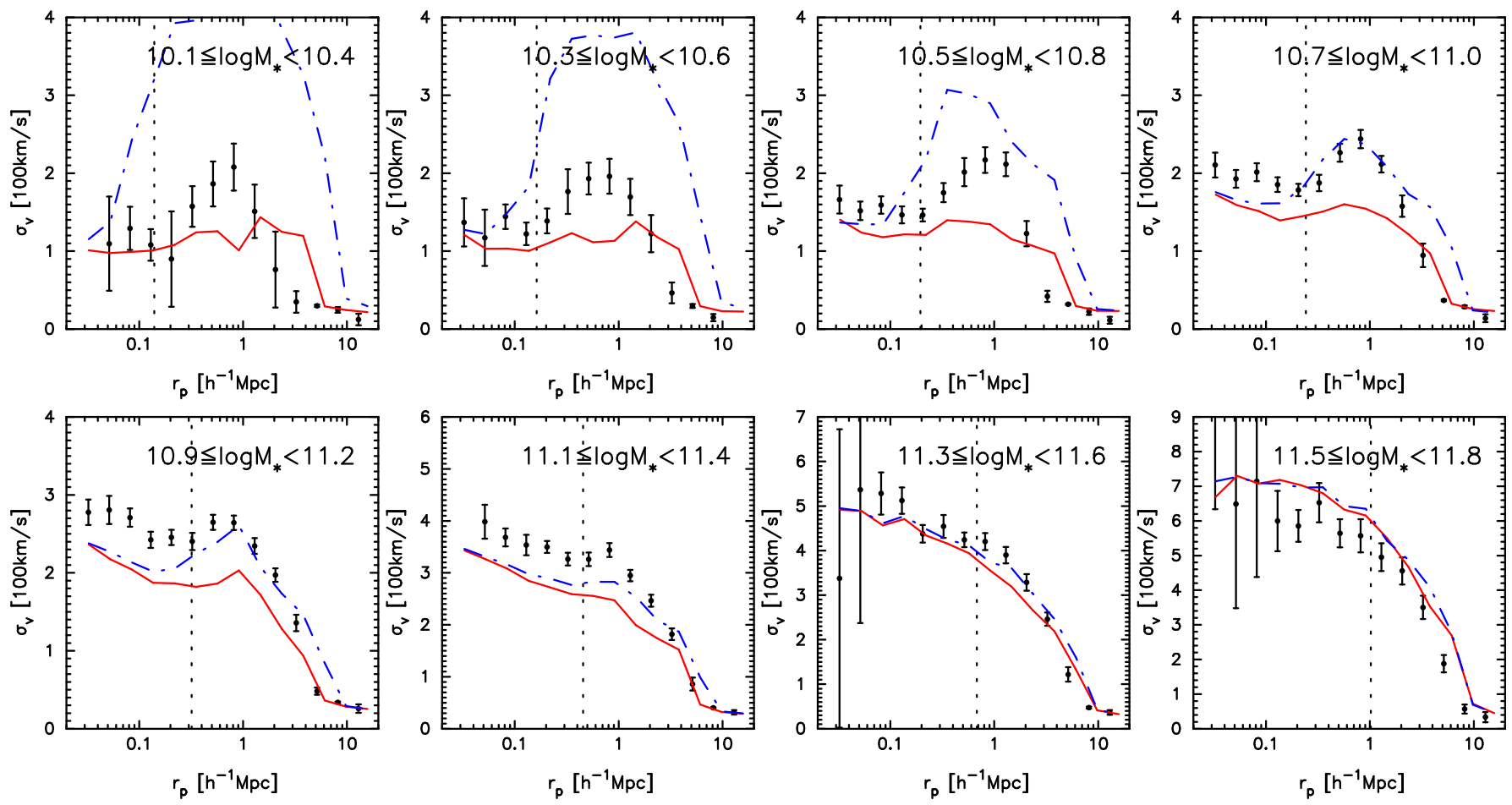

FIG. 5. - The velocity dispersion profile measured from the SDSS data are plotted in black symbols with error bars for some of our group subsamples, and are compared with the measurements from dark matter subhalo catalogs before (blue dash-dotted lines) and after (red solid lines) 'blending' close halos (see the text for details). The vertical lines indicate the virial radii corresponding to the halo masses listed in Table 1. 
observation obtained above.

We take all the halos from the $z=0$ outputs of our simulations, and we assign a stellar mass to each halo by applying the commonly-applied subhalo abundance matching model (SHAM; Vale \& Ostriker 2004; Conroy et al. 2006; Shankar et al. 2006; Conroy et al. 2007a; Baldry et al. 2008; Moster et al. 2010; Guo et al. 2010; Neistein et al. 2011b,a; Yang et al. 2012). The model assumes that each halo has a galaxy at its center and the stellar mass of a galaxy is an increasing function of the maximum mass ever attained by its halo. In practice, we obtain the relationship between dark halo mass $M_{200}$, as defined in $\S 2.3$, and the stellar mass $M_{*}$ of the central galaxy by matching the number density of dark matter halos with mass above $M_{200}$ with the number density of galaxies with stellar mass above $M_{*}$. For this we have used the stellar mass function of galaxies in the local Universe measured by Li \& White (2009) from the SDSS/DR7 galaxy sample, corrected in Guo et al. (2010) using "model" magnitudes. For the analysis in this subsection we assume there is no scatter in the $M_{200}-M_{*}$ relationship, and we will take into account the effect of the scatter in later analyses.

We note that, as pointed out recently by Yang et al. (2012), one problem of the current SHAMs is that they generally neglect the evolution of the central galaxy-host halo relation (see also Neistein et al. 2011a) and the different stripping/disruption of satellite galaxies with respect to the subhalos. Realistically, satellite galaxies were accreted at higher redshifts and should have experienced different evolutionary processes from the centrals. Nevertheless, as shown in Yang et al. (2012), current high redshift observations do imply that there is little evolution of the central-host halo relation at low redshifts $(z \lesssim 1)$. Moreover, the central-host halo relation for halos with masses $\gtrsim 10^{12} M_{\odot}$ is found to be insensitive to these caveats. Thus the approach of SHAMs is quite safe for our study where the galaxies are at $z \sim 0$ and are hosted by dark halos with mass well above $10^{12} M_{\odot}$ (see below).

The galaxy groups used here are identified using the so called halo-based group finder, where galaxies are grouped according to their common dark matter halos following the NFW profiles, i.e., by assuming the halos to be Spherical Overdensity (SO) structures. Such kind of SO method can be quite different from the FoF algorithm where each halo/group is a self-bound virialized system. On the other hand, the FoF halos might have bridging effect - in some cases, two or more subhalos that are well separated in space are associated with a common FoF halo and only one of them (usually the most massive one) is classified as the central subhalo. In order to roughly take into account the difference between the FoF and SO halo finding algorithm, we have found out all the satellite subhalos that are located well beyond the virial radius of their central subhalo $\left(R_{200, c}\right)$, by requiring that $d_{c s}>R_{200, c}+R_{200, s}$, where $d_{c s}$ is the distance from the central subhalo and $R_{200, s}$ is the virial radius of the satellite subhalo at the epoch when it was last the central object of its host halo (see $\S 2.3$ ). We reidentify such satellite subhalos as central subhalos and include them into the central galaxy subsamples when performing the analysis below.
We have estimated both the cross-correlation functions and velocity dispersion profiles for the halo catalog. First, the central subhalos are divided into a set of subsamples according to stellar mass, with the same mass intervals as listed in Table 1 for the real subsamples. Next, each subsample is cross-correlated with the full catalog to compute a redshift-space crosscorrelation function, $\xi^{(s)}\left(r_{p}, \pi\right)$, as well as a projected cross-correlation function, $w_{p}\left(r_{p}\right)$. We have followed the distant-observer approximation, defining the $x-y$ plane of the simulation box as the 'sky' and the $z$-axis as the line of sight. The difference in the $z$-axis position between two galaxies and the difference in their peculiar velocity along the same axis combine to give a line-ofsight separation, $\pi$, thus including the redshift distortions in the computation of $\xi^{(s)}\left(r_{p}, \pi\right)$. We should point out that we have added a random component to the peculiar velocity of the central subhalos, assuming it to follow a Gaussian distribution function with a $1 \sigma$ width of $0.25 V_{200}$, where $V_{200}$ is the virial velocity of the halo. This takes into account the fact that the central galaxies of dark halos are not strictly at rest, but move relative to the halo center with a velocity that is $20-30 \%$ the virial velocity (Diaferio et al. 1999; Berlind et al. 2003; Yoshikawa et al. 2003; van den Bosch et al. 2005; see also Skibba et al. 2011 and references therein). Finally, the velocity dispersion profiles are determined by applying the $\xi^{(s)}\left(r_{p}, \pi\right)$ model in Eqn. (8) to the $\xi^{(s)}\left(r_{p}, \pi\right)$ measured from the halo catalogs, in exactly the same way as adopted for the real samples.

In Fig. 5 we plot in blue dash-dotted lines the velocity dispersion profiles measured from one of our simulations, the one with $\sigma_{8}=0.85$, and compare these with measurements from the SDSS data (symbols with error bars). At all masses, the model and the data show very similar profiles over the full range of $r_{p}$ probed. In particular, the bump at around $1 \mathrm{Mpc}$ is seen clearly in the model. For systems with intermediate to high masses $\left(M_{*} \gtrsim 5 \times 10^{10} M_{\odot}\right)$, the bump in the model is comparable to that observed, in both amplitude and shape. At lower masses, the bump is stronger in the model. As pointed out above, one possible reason behind the bump in the observed profiles is the contamination from neighboring halos in which the peculiar motions of galaxies can contribute significantly to the velocity dispersion measurement of the system in question. To test this hypothesis, we have done a simple experiment in which we exclude all the halos from our central galaxy subsamples that are close to a neighboring larger halo, with the distance between the two halos smaller than the sum of their virial radii. The resulting velocity dispersion profiles are plotted in red solid lines in Fig. 5. As can be seen, the bumps at around $1 \mathrm{Mpc}$ become very weak or disappear, as expected.

The change in the velocity dispersion profile is strongest at the lowest masses where the bump is no longer significant after the close halos are 'blended'. We have played around with different distance limits for blending the halos, and found that the bump becomes stronger for smaller blending radii. This can be understood from the fact that a smaller blending radius will include more satellite subhalos located in the inner region of FoF halos where the contamination in velocity is 
more serious. At the highest masses $\left(M_{*} \gtrsim 2 \times 10^{11} M_{\odot}\right)$, the velocity dispersion profile is essentially unchanged, reflecting the fact that the majority of the high-mass galaxies in the local Universe are central galaxies of dark matter halos. At these masses, the satellite fraction is found to be as low as $\sim 10 \%$ by galaxy-galaxy lensing analyses of the SDSS data (Mandelbaum et al. 2006), as well as the SDSS-based halo occupation models (Cooray 2006; Tinker 2007; van den Bosch et al. 2007).

It is encouraging that the 'deblending' effect doesn't change the velocity dispersion profile within the virial radius for all the stellar mass subsamples, except the lowest ones with $\log M_{*} / M_{\odot} \lesssim 10.3$. This shows that, although the profile beyond the virial radius is somewhat uncertain due to the contamination from neighboring larger systems, the inner part can be well determined with the current data and methodology. Therefore, in what follows we limit our analyses to masses above $\log M_{*} / M_{\odot}=10.3$ and focus on velocity dispersions within the virial radius, and we use the original subhalos identified by our HBT subhalo finder, without further mimicking the group finding algorithm of Yang et al. (2007).

\subsection{Constraining density fluctuation parameter $\sigma_{8}$ and the stellar mass vs. halo mass relation}

The velocity dispersion of galaxies reflects the action of the local gravitational field, and so should provide independent constraints on the connection between galaxies and dark matter halos. In addition, the relationship between galaxy velocity dispersion and mass is expected to depend on the density fluctuation parameter $\sigma_{8}$. This is simply because $\sigma_{8}$ largely determines the abundance of the most massive halos, thus the velocity dispersion of the most massive galaxies that are hosted by these halos. Here, we extend the analysis in the previous subsection and show that the $\sigma_{8}$ parameter and the stellar mass vs. halo mass relation can be simultaneously constrained using our estimates of velocity dispersion obtained above.

To the end we use all the simulations with different $\sigma_{8}$ values. We also allow the central galaxy mass of halos of given dark matter mass to have a certain amount of dispersion. Following the standard practice, we assume the dispersion in $\log M_{*}$ to be Gaussian and independent of halo mass, with rms values ranging from $\sigma_{m s}=0$ to 0.2 dex. The rms values exceeding about 0.2 dex are excluded because they are inconsistent with the steep high-mass falloff of the stellar mass function of Li \& White (2009).

In Fig. 6 we plot the velocity dispersion profiles estimated from the subhalo catalogs for four of the stellar mass intervals (panels from left to right), and for models with different dispersions in the stellar mass vs. halo mass relation (from top to bottom) and with different $\sigma_{8}$ values (the colorful lines). The observational results are plotted in symbols with error bars for comparison. The virial radii are indicated in each panel with the vertical lines, which are given by Eqn.(1) for the median halo mass of subhalos in a given stellar mass subsample. Overall, the velocity dispersions within the virial radii depend on both $\sigma_{8}$ and $\sigma_{m s}$, but the behavior differs with stellar mass. At low masses, the velocity dispersion increases with increasing $\sigma_{m s}$, but shows very little dependence on $\sigma_{8}$. At high masses, the velocity dispersion depends on both parameters with a stronger trend with $\sigma_{8}$. The velocity dispersion is higher for systems with fixed mass and with fixed $\sigma_{m s}$ if $\sigma_{8}$ is larger, well consistent with the naive expectation as discussed above. The weak dependence on $\sigma_{8}$ at the low-mass end is very encouraging, in the sense that the degeneracy between $\sigma_{8}$ and $\sigma_{m s}$ as seen at high masses can be effectively broken, allowing the two parameters to be constrained simultaneously.

For each stellar mass interval, we determined an average velocity dispersion using the measurements falling in the range $0.3 R_{200}<r_{p}<R_{200}$. We exclude the innermost part within $0.3 R_{200}$ considering the facts that the velocity dispersion profile slightly increases at the smallest radii for massive systems (see Fig. 4) and this is not seen in the models at similar masses, and that we don't have velocity dispersion measurements on scales below $0.3 R_{200}$ for low mass systems (again see Fig. 4). The former fact is consistent with previous findings that the inner slope determined from gravitational lensing analyses is steeper than that expected from the NFW profile, implying that baryonic processes have modified the inner profiles (e.g. Koopmans et al. 2006). The average velocity dispersions obtained from the SDSS data are listed in Column 5 of Table 1. In Fig. 7 we show the average velocity dispersion $\sigma_{v}$ as a function of stellar mass. Results from the SDSS data are plotted in symbols with error bars and are repeated in every panel. These are compared with results from the various models which have different values of $\sigma_{8}$ (panels from left to right) and $\sigma_{m s}$ (different lines in each panel). The degeneracy between $\sigma_{8}$ and $\sigma_{m s}$, as well as the capability of the $\sigma_{v}-M_{*}$ relation for breaking the degeneracy, are seen more clearly in this figure. At low masses, the velocity dispersion shows strong dependence on $\sigma_{m s}$. The dependence on $\sigma_{m s}$ is much weaker at high masses, particularly at $\sim 2 \times 10^{11} M_{\odot}$ where the velocity dispersion is essentially determined by $\sigma_{8}$ alone. The data at these masses clearly suggest that an intermediate value of $\sigma_{8}=0.85$ works better than either higher or lower values.

In order to have a more quantitative constraint on both $\sigma_{8}$ and $\sigma_{m s}$, we have performed the analysis for more models by varying the two parameters, with $\sigma_{m s}$ ranging from 0 to 0.2 dex with a step size of 0.01 , and $\sigma_{8}$ ranging from 0.75 to 0.95 with a step size of 0.01 . For given $\sigma_{m s}$, we estimate velocity dispersions for models of different $\sigma_{8}$ by interpolating the $\sigma_{v}-M_{*}$ relation obtained from the three simulations, assuming the velocity dispersion at fixed stellar mass to scale linearly with $\sigma_{8}$. This assumption is reasonably true considering the following two facts. First, the change in velocity dispersion with $\sigma_{8}$ is fairly small, as can be seen from Fig. 7. Second, when obtaining the number density of galaxy clusters through the Press \& Schechter (1974) formalism, Mo et al. (2010, see their Eqns. 7.75 and 7.76) found that $\sigma_{8}$ scales with cluster mass as $\sigma_{8} \propto M^{\beta / 2} \Omega_{m}^{-\beta / 3}$, where $\beta \approx 0.6+0.8 h \Omega_{m}$ for CDM-type power spectra with index $n=1$, leading to a nearly linear relation between $\sigma_{8}$ and velocity dispersion $\sigma_{v}$ for the assumed cosmology, $\sigma_{v} \propto \sigma_{8}^{0.9}$, given that the mass is roughly proportional to $\sigma_{v}^{3}$.

We compare the velocity dispersions of each model to the SDSS measurements, and define the best-fitting model to be the one giving a minimum $\chi^{2}$ computed as follows:

$$
\chi^{2}\left(\sigma_{8}, \sigma_{m s}\right)=\mathbf{X}^{\mathbf{T}} \mathbf{C}^{-1} \mathbf{X}
$$



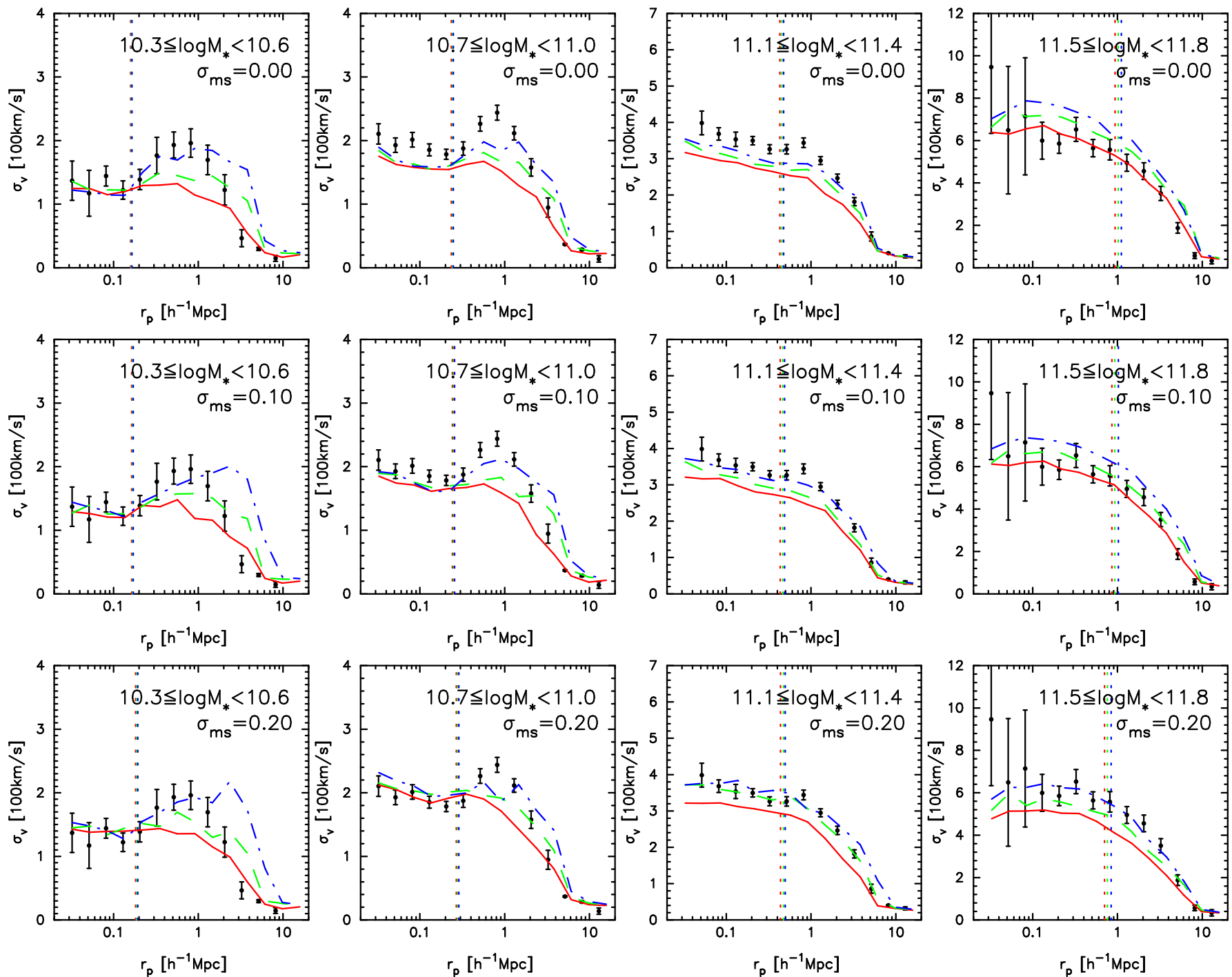

FIG. 6.- Velocity dispersion profiles estimated from subhalo catalogs for different stellar mass intervals (panels from left to right), and for models with different dispersions in the stellar mass vs. halo mass relation (panels from top to bottom) and with different density fluctuation parameter: $\sigma_{8}=0.75$ (red solid line), 0.85 (green dashed line) and 0.95 (blue dash-dotted line). The estimates from the SDSS data are plotted in symbols with error bars for comparison. The virial radii from the models are indicated by the vertical lines.
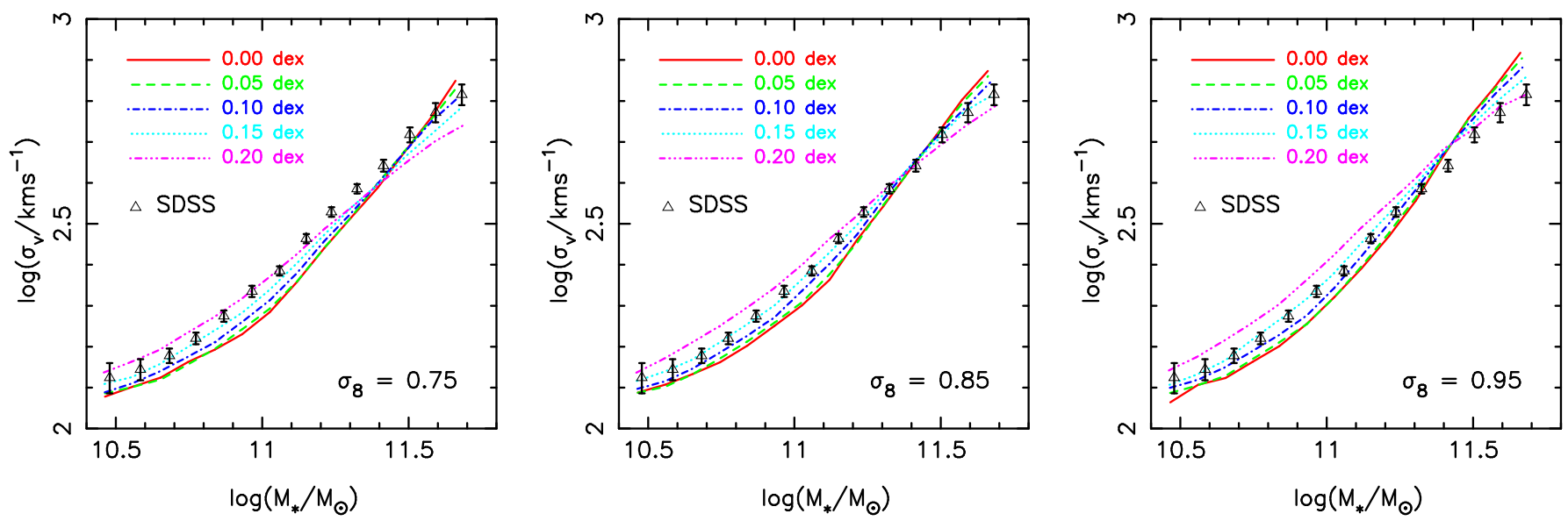

FIG. 7.- Velocity dispersion averaged over $0.3 R_{200}<r_{p}<R_{200}$ is plotted as a function of central galaxy mass. Results from the SDSS data are plotted in symbols with error bars and are repeated in every panel. Results from the models are plotted in different lines, which have different values of $\sigma_{8}$ (panels from left to right) and $\sigma_{m s}$ (different lines in each panel). 


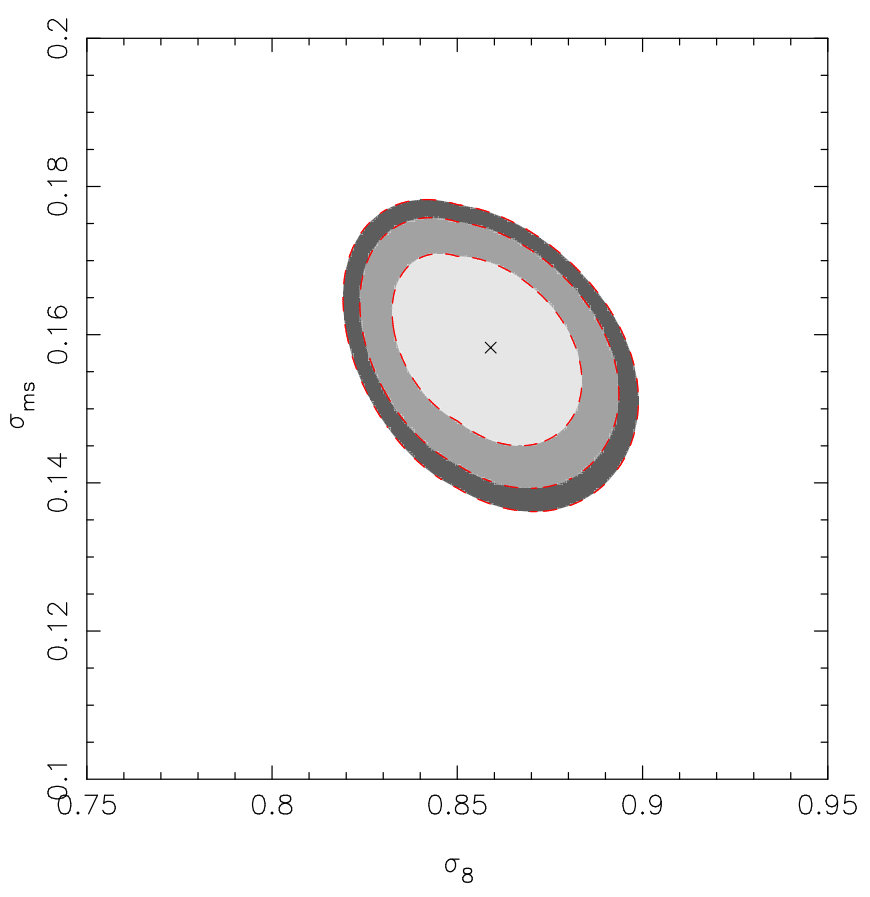

FIG. 8. - $\chi^{2}$, defined by Eqn.(14) and derived by comparing the average velocity dispersion within the virial radius of dark matter halos as predicted by the subhalo abundance matching model and as measured from groups of different central galaxy masses from the SDSS data, is plotted with respect to the minimum value $\chi_{\min }^{2}$ (indicated by an ' $\mathrm{x}$ '), in the grid of the two model parameters, $\sigma_{8}$ and $\sigma_{m s}$. The $68.3 \%, 90 \%$ and $95.4 \%$ confidence levels are plotted as red dashed lines, and the area between every two neighboring levels is filled with shaded regions.

Here, $\mathbf{X}=\left\{X_{j}\right\}(j=1, \ldots, m)$ is an $m \times 1$ vector with

$$
X_{j}=\sigma_{v, j}^{\text {model }}-\sigma_{v, j}^{S D S S},
$$

where $m$ is the number of stellar mass subsamples, $\sigma_{v, j}^{S D S S}$ is the velocity dispersion measured for the $j$ th subsample from the SDSS and $\sigma_{v, j}^{\text {model }}$ is the result for the $j$ th subsample from the model. The $m \times m$ matrix $\mathbf{C}=\left\{C_{i j}\right\}(i, j=1, \ldots, m)$ is the covariance matrix of the measurements from the 100 bootstrap samples constructed from the SDSS data, given by

$$
C_{i j}=\frac{1}{n}\left[\sum_{k=1}^{n}\left(\sigma_{v, k i}^{b o o t}-\sigma_{v, i}^{S D S S}\right)\left(\sigma_{v, k j}^{b o o t}-\sigma_{v, j}^{S D S S}\right)\right],
$$

where $n=100$ is the number of bootstrap samples, and $\sigma_{v, k i}^{b o o t}$ is the measurement for the $i$ th stellar mass interval from the $k$ th bootstrap sample.

Fig. 8 plots the contours of $\Delta \chi^{2}=\chi^{2}-\chi_{\min }^{2}$ in the grid of the two parameters. The 1,2 and $3 \sigma$ confidence regions, computed for $m=14$ and 2 parameters, are indicated using the dashed lines plus shaded regions. The minimum $\chi_{\min }^{2}$ appears at $\sigma_{8}=0.86$ and $\sigma_{m s}=0.16$ with $\chi^{2} /$ d.o. $f=0.4$, indicating that the fit is acceptable. There is a mild degeneracy between the two parameters in the sense that the models with smaller $\sigma_{8}$ and larger $\sigma_{m s}$ and the models with larger $\sigma_{8}$ and smaller $\sigma_{m s}$ can both provide a reasonable fit to the data. While the minimum is the preferred solution at the $1 \sigma$ level, there is an elliptical degeneracy region extending to $(0.82$,

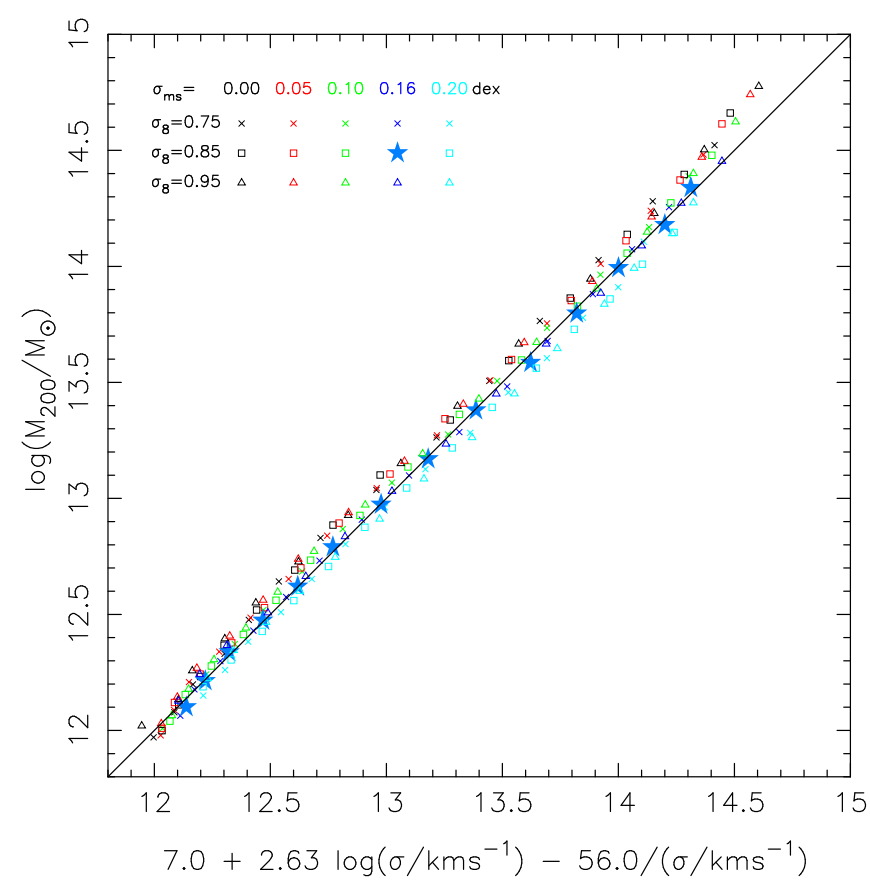

FIG. 9.- Median halo mass $M_{200}$ for central subhalos with given stellar mass is plotted as a function of the velocity dispersion measured using our methodology, for the models with different values of $\sigma_{8}$ and $\sigma_{m s}$. The black solid line is the relation obtained by fitting to the result from the model with $\sigma_{8}=0.85$ and $\sigma_{m s}=0.15$ (plotted as big stars), and is adopted as our calibration for estimating halo masses from velocity dispersion measurements. The calibrated estimator is indicated at the bottom of the figure.

$0.17)$ and $(0.90,0.14)$, that are allowed at $2-3 \sigma$ level. Our constraint on $\sigma_{m s}$ is in good agreement with previous studies which also found that the dispersion in stellar mass at fixed halo mass is $~ 0.13-0.20$ dex (e.g. Wang et al. 2006, 2007; Yang et al. 2009; Behroozi et al. 2010; Moster et al. 2010; More et al. 2011; Yang et al. 2012).

Our constraint on $\sigma_{8}$ is slightly larger than the result from the Wilkinson Microwave Anisotropy Probe 7 (WMAP7) and low redshift supernova and baryon acoustic oscillation data (Komatsu et al. 2011), which was $\sigma_{8}=0.816$. We note that this difference can be largely explained by the slightly different matter density parameter, which was $\Omega_{m}=0.275$ in Komatsu et al. (2011) and is $\Omega_{m}=0.268$ in our simulations. This implies that our preferred value would be shifted to $\sigma_{8} \approx 0.84$ for the WMAP7 matter density parameter, thus within the $1 \sigma$ level of the WMPAP7 result.

\subsection{Estimating galaxy halo masses}

The velocity dispersion is caused by the local gravitational field and so provides a direct measure of the dark halo mass of galaxies. We use our simulations to calibrate the relationship between the velocity dispersion measured using our methodology and the halo mass for central subhalos of different stellar masses. As plotted in Fig. 9, the best-fitting relation is

$$
\begin{aligned}
\log \left(M_{200} / M_{\odot}\right)= & 7.0+2.63 \log \left(\sigma_{v} / \mathrm{kms}^{-1}\right) \\
& -56.0 /\left(\sigma_{v} / \mathrm{kms}^{-1}\right),
\end{aligned}
$$



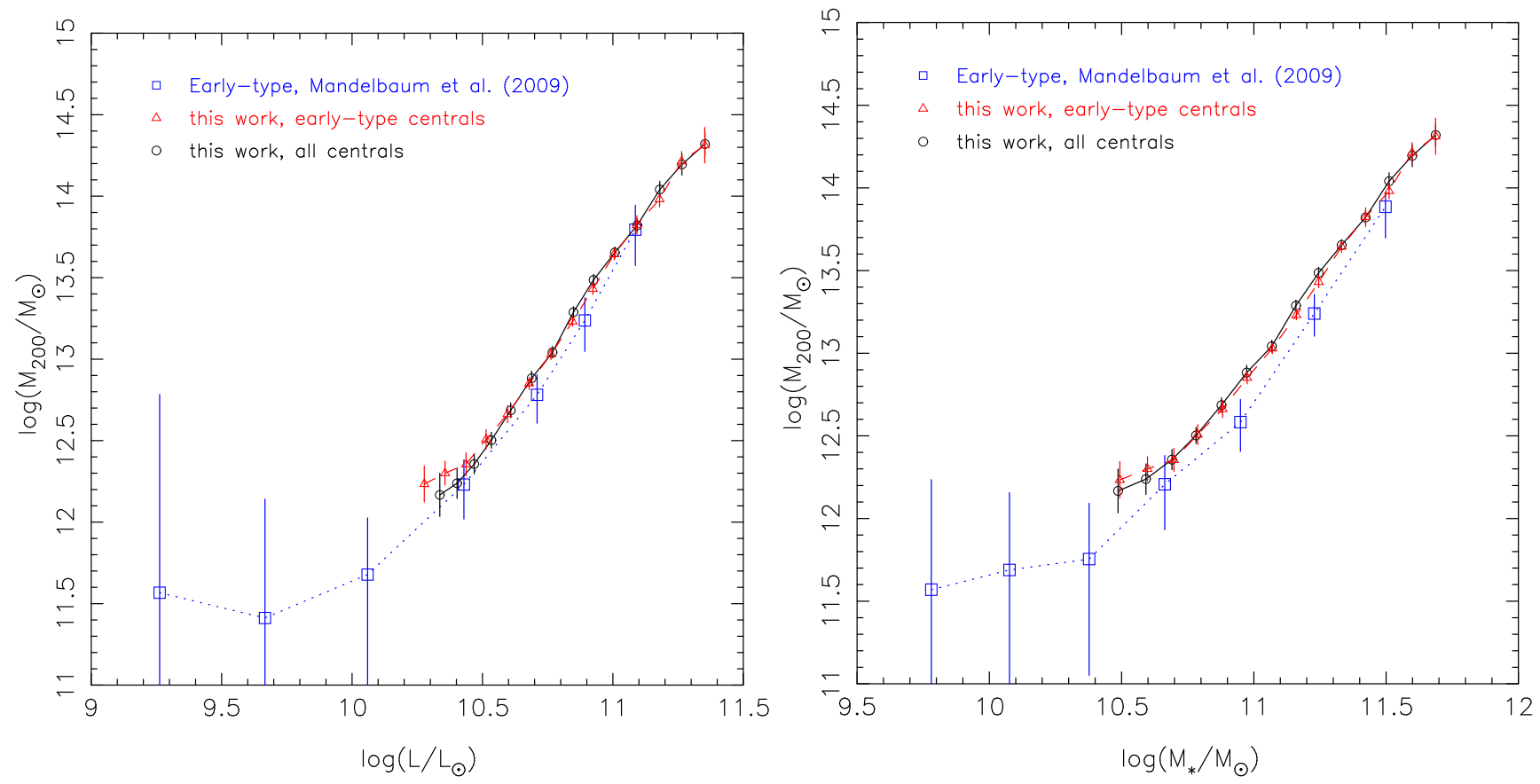

FIG. 10.- Dark matter halo mass as function of galaxy luminosity (left-hand panel) and stellar mass (right-hand panel). Black circles and red triangles are estimated from the velocity dispersion measurements, for all the central galaxies in our group catalog and for the subset of early-type centrals, respectively. Blue squares are the result obtained by Mandelbaum et al. (2006) by stacking the gravitational lensing signals of the early-type central galaxies in an earlier SDSS data release.

obtained using the subhalo abundance matching model applied to the simulation with $\sigma_{8}=0.85$ and with the dispersion parameter $\sigma_{m s}=0.16$. As shown above, this model provides the best match to the SDSS data in the stellar mass versus velocity dispersion relation.

We apply the halo mass estimator in Eqn.(17) to our measurements of velocity dispersions. The resulting halo masses are listed in the last column of Table 1 and are plotted in Fig. 10 as a function of both luminosity (left-hand panel) and stellar mass (right-hand panel). For comparison, we also plot the results obtained by Mandelbaum et al. (2006) from stacking the gravitational lensing signals from the SDSS data. We note that about $90 \%$ of our central galaxies are early-type galaxies, which are defined in practice to have the parameter frac_deV $\geq 0.5$ from the SDSS PнотO pipeline. In order to make a fair comparison, we have measured the velocity dispersions for groups with early-type centrals and plot the results as red triangles in Fig. 10. The result doesn't change much when compared to that of the full sample. The halo masses of early-type centrals presented in Mandelbaum et al. (2006) are plotted as open squares. As can be seen, the halo masses estimated from galaxy velocity dispersions agree pretty well with those from gravitational lensing signals, over the full luminosity range $\left(2 \times 10^{10} L_{\odot} \lesssim L \lesssim 1.5 \times 10^{11} M_{\odot}\right)$ where the halo mass estimates are available from both studies. Note that we have shifted the halo masses of Mandelbaum et al. (2006) by about -0.1 dex in order to take into account the different halo mass definitions adopted in the two studies.

The agreement becomes slightly worse when the halo mass is plotted as a function of stellar mass, as shown in the right-hand panel of Fig. 10. In this case our halo mass is systematically higher than that from Mandelbaum et al. (2006) by about 50\% at intermediate to low masses. The stellar masses used in Mandelbaum et al. (2006) were estimated by Kauffmann et al. (2003) based on the SDSS spectroscopy, while those in this study are based on the photometric properties. Therefore, for this comparison in Fig. 10, we have shifted the former by about 0.1 dex in stellar mass; the correction factor was obtained in Appendix A of Li \& White (2009) using a large sample of galaxies for which both stellar mass estimates are available (see also fig.17 of Blanton \& Roweis 2007). As can be seen in Li \& White (2009), the difference between the two stellar masses depends on stellar mass in a complicated way with varying scatter. Thus, a constant correction cannot fully get rid of the difference, and the larger discrepancy between our results and those of Mandelbaum et al. (2006) as expressed in terms of stellar mass must be partially (if not totally) due to this effect.

\section{SUMMARY AND DISCUSSION}

Using a large catalog of galaxy groups selected from the Sloan Digital Sky Survey (SDSS) data release 7 (DR7) by Yang et al. (2007), we have derived the velocity dispersion profiles for groups with different masses of central galaxies, by modelling the redshift distortions in groupgalaxy cross-correlation function. Our main results can be summarized as follows.

- An Navarro et al. (1997) profile on small scales plus a biased version of the linear mass autocorrelation function can well describe the observed $w_{p}\left(r_{p}\right)$ over all the scales probed $\left(15 \mathrm{kpc}<r_{p}<30 \mathrm{Mpc}\right)$, thus providing an accurate determination of the realspace cross-correlation function $\xi_{c g}(r)$. This has 
been well expected from theoretical studies of halomass and galaxy-mass cross-correlation functions (e.g. Hayashi \& White 2008).

- The velocity dispersion within virial radius $\left(R_{200}\right)$ shows a roughly flat profile, with a slight increase at the smallest radii $\left(\lesssim 0.3 R_{200}\right)$. This is consistent with the theoretical expectation that baryonic condensation has modified the inner profile.

- The average velocity dispersion within the virial radius, $\sigma_{v}$, is a strongly increasing function of central galaxy mass, ranging from $\sim 130 \mathrm{kms}^{-1}$ for the lowest-mass groups in our sample which have central galaxy masses of $M_{*} \sim 3 \times 10^{10} M_{\odot}$, up to $\sim 650 \mathrm{kms}^{-1}$ for the highest-mass systems with $M_{*} \sim 5 \times 10^{11} M_{\odot}$. The mass dependence is more remarkable at higher masses.

We have extended our analysis of the SDSS data to a set of $N$-body simulations with the concordance $\Lambda$ cold dark matter $(\Lambda \mathrm{CDM})$ cosmology but different values of the density fluctuation parameter $\sigma_{8}$. We have assigned a stellar mass to each of our subhalos under the plausible hypothesis that the stellar mass of central galaxies is an increasing function of the dark matter mass of their host halos, usually called subhalo abundance matching model in the literature, and measured the velocity dispersion profile for the subhalos in different stellar mass intervals. By comparing the results to measurements from the SDSS data, we have shown that the $\sigma_{v}-M_{*}$ relation obtained from the data provides stringent constraints on both the $\sigma_{8}$ parameter and $\sigma_{m s}$, the dispersion in $\log M_{*}$ of central galaxies at fixed halo mass. Our best-fitting model suggests that $\sigma_{8}=0.86 \pm 0.03$ and $\sigma_{m s}=0.16 \pm 0.03$. The latter is in very good agreement with previous studies where $\sigma_{m s}$ was inferred from semi-analytic catalogs of galaxy formation models (e.g. Wang et al. 2006, 2007), from group catalogs (Yang et al. 2009), from satellite kinematics analysis (More et al. 2011), from clustering analysis (Moster et al. 2010), and from subhalo abundance matching (Behroozi et al. 2010; Guo et al. 2010; Yang et al. 2012).

Our velocity dispersion measurements also provide a direct measure of the dark matter mass of the host halos for central galaxies of different luminosities and stellar masses, which are in good agreement with the results obtained by Mandelbaum et al. (2006) from stacking the gravitational lensing signals of the SDSS galaxies. The results also broadly agree with recent estimates by many other authors (e.g. van den Bosch et al. 2004; Yang et al. 2007; More et al. 2009b; Guo et al. 2010; More et al. 2011), though with varying degrees of subtle discrepancies due to different methodologies and date sets.

It is encouraging to see that the velocity dispersion as a function of central galaxy mass as measured in our study is able to constrain the density fluctuation parameter $\sigma_{8}$, although the preferred value is slightly higher than that from the most recent result from WMAP7 (Komatsu et al. 2011). We suspect that this difference can be explained if the effect of the different matter density parameters adopted in the two studies is considered. It has been recently emphasized that the redshift-space distortion on very large scales (near or beyond the baryon acoustic oscillation scale), expected to be well detected in next-generation redshift survey like the BigBOSS experiment (Schlegel et al. 2011), will provide powerful constraints on the growth factor of structure and the physical properties of dark energy (e.g. Okumura \& Jing 2011; Samushia et al. 2012). Our study suggests that the redshift distortions on galaxy and group scales provide complementary information useful for constraining cosmological parameters. When combined with more observational measurements like the autocorrelation function and pairwise velocity dispersion of galaxies as functions of their physical properties (e.g. Zehavi et al. 2005; Li et al. 2006a,b), our results should be able to provide more accurate and reliable constraints on $\sigma_{8}$. We will come back to this point in future studies.

There is still room to improve our study. First, we have assumed isotropic distributions for the peculiar velocity and velocity dispersion of satellite galaxies and this is roughly, but not exactly correct. Models incorporated with radial anisotropy might provide better determinations of the velocity dispersions. Using red, early-type galaxies instead of the whole population as the tracer may also help, as they are more likely consistent with isotropic orbits than are blue and late-type galaxies (e.g. Carlberg et al. 1997c; Biviano \& Katgert 2004). Second, we have used groups with three or more member galaxies and assumed that our determinations of velocity dispersion are insensitive to the richness limit of the group sample. Larger and deeper surveys in future would allow us to test this assumption and improve our method. Third, previous studies found that Brightest Cluster Galaxies (BCG) may be not at the true center of their host halo (e.g. Skibba et al. 2011), and this effect could also introduce some sort of systematics. Fortunately, this is unlikely a significant issue for us, as such offsets seem to be small in general (a recent study by von der Linden et al. 2012 found a median value of $20 \mathrm{kpc}$ in the ffsets between X-ray centroids and BCGs in clusters). Fourth, we have assumed the dispersion in stellar mass is independent of halo mass and this might be tested and improved via semi-analytic galaxy formation models or halo occupation distribution models. Finally, our work is limited to relatively large systems with $M_{*} \gtrsim 3 \times 10^{10} M_{\odot}$ and could be extended to lower masses using deeper surveys. Previous studies of the pairwise velocity dispersion (PVD) of galaxies revealed that the relative velocities between galaxies exhibit a well-defined minimum at intermediate luminosities/masses, and both fainter and brighter galaxies have very high velocities, indicative of massive halos of cluster size (Jing \& Börner 2004; Li et al. 2006a). It would be interesting to directly measure the halo mass for such faint galaxies by applying our methodology to groups of lower masses, e.g. down to $\sim 6 \times 10^{9} M_{\odot}$ using the Galaxy And Mass Assembly survey (GAMA; Driver et al. 2009).

We are grateful to the referee for helpful comments. CL is grateful to Qi Guo, Donghai Zhao, Simon White, Raul Angulo and Ying $\mathrm{Zu}$ for helpful discussion, and acknowledges the support of the 100-Talent Program of Chinese Academy of Sciences (CAS), Shanghai Pujiang Programme (no. 11PJ1411600) and the exchange program between Max Planck Society and CAS. This work 
is sponsored by NSFC (11173045, 10878001, 11033006, 11121062, 10925314, 11128306) and the CAS/SAFEA International Partnership Program for Creative Research Teams (KJCX2-YW-T23).

Funding for the SDSS and SDSS-II has been provided by the Alfred P. Sloan Foundation, the Participating Institutions, the National Science Foundation, the U.S. Department of Energy, the National Aeronautics and Space Administration, the Japanese Monbukagakusho, the Max Planck Society, and the Higher Education Funding Council for England. The SDSS Web Site is http://www.sdss.org/. The SDSS is managed by the Astrophysical Research Consortium for the Participating Institutions. The Participating Institutions are the American Museum of Natural History, Astrophysi- cal Institute Potsdam, University of Basel, University of Cambridge, Case Western Reserve University, University of Chicago, Drexel University, Fermilab, the Institute for Advanced Study, the Japan Participation Group, Johns Hopkins University, the Joint Institute for Nuclear Astrophysics, the Kavli Institute for Particle Astrophysics and Cosmology, the Korean Scientist Group, the Chinese Academy of Sciences (LAMOST), Los Alamos National Laboratory, the Max-Planck-Institute for Astronomy (MPIA), the Max-Planck-Institute for Astrophysics (MPA), New Mexico State University, Ohio State University, University of Pittsburgh, University of Portsmouth, Princeton University, the United States Naval Observatory, and the University of Washington.

\section{REFERENCES}

Abazajian, K. N., Adelman-McCarthy, J. K., Agüeros, M. A., et al. 2009, ApJS, 182, 543

Baldry, I. K., Glazebrook, K., \& Driver, S. P. 2008, MNRAS, 388, 945

Barrow, J. D., Bhavsar, S. P., \& Sonoda, D. H. 1984, MNRAS, 210, 19P

Becker, M. R., McKay, T. A., Koester, B., et al. 2007, ApJ, 669, 905

Behroozi, P. S., Conroy, C., \& Wechsler, R. H. 2010, ApJ, 717, 379

Bell, E. F., McIntosh, D. H., Katz, N., \& Weinberg, M. D. 2003, ApJS, 149, 289

Berlind, A. A., Weinberg, D. H., Benson, A. J., et al. 2003, ApJ, 593,1

Biviano, A., \& Katgert, P. 2004, A\&A, 424, 779

Blanton, M. R., Lupton, R. H., Schlegel, D. J., et al. 2005, ApJ, 631,208

Blanton, M. R., \& Roweis, S. 2007, AJ, 133, 734

Blanton, M. R., Brinkmann, J., Csabai, I., et al. 2003, AJ, 125, 2348

Blumenthal, G. R., Faber, S. M., Flores, R., \& Primack, J. R. 1986, ApJ, 301, 27

Brainerd, T. G., \& Specian, M. A. 2003, ApJ, 593, L7

Cabré, A., \& Gaztañaga, E. 2009, MNRAS, 396, 1119

Carlberg, R. G., Yee, H. K. C., \& Ellingson, E. 1997a, ApJ, 478, 462

Carlberg, R. G., Yee, H. K. C., Ellingson, E., et al. 1996, ApJ, 462,32

Carlberg, R. G., Yee, H. K. C., Morris, S. L., et al. 2001, ApJ, 552,427

Carlberg, R. G., Yee, H. K. C., Ellingson, E., et al. 1997b, ApJ, 485, L13

- $1997 \mathrm{c}$, ApJ, 476, L7

Chabrier, G. 2003, PASP, 115, 763

Chen, J., Kravtsov, A. V., Prada, F., et al. 2006, ApJ, 647, 86

Colless, M., Dalton, G., Maddox, S., et al. 2001, MNRAS, 328, 1039

Conroy, C., Wechsler, R. H., \& Kravtsov, A. V. 2006, ApJ, 647, 201

. 2007a, ApJ, 668, 826

Conroy, C., Prada, F., Newman, J. A., et al. 2007b, ApJ, 654, 153

Cooray, A. 2006, MNRAS, 365, 842

Cooray, A., \& Sheth, R. 2002, Phys. Rep., 372, 1

Croft, R. A. C., Dalton, G. B., \& Efstathiou, G. 1999, MNRAS, 305,547

Davis, M., Efstathiou, G., Frenk, C. S., \& White, S. D. M. 1985, ApJ, 292, 371

Davis, M., \& Peebles, P. J. E. 1983, ApJ, 267, 465

Diaferio, A., Kauffmann, G., Colberg, J. M., \& White, S. D. M. 1999, MNRAS, 307, 537

Driver, S. P., Norberg, P., Baldry, I. K., et al. 2009, Astronomy and Geophysics, 50, 050000

Erickson, L. K., Gottesman, S. T., \& Hunter, Jr., J. H. 1987, Nature, 325,779

Fisher, K. B., Davis, M., Strauss, M. A., Yahil, A., \& Huchra, J. P. 1994, MNRAS, 267, 927

Gnedin, O. Y., Kravtsov, A. V., Klypin, A. A., \& Nagai, D. 2004, ApJ, 616, 16

Guo, Q., White, S., Li, C., \& Boylan-Kolchin, M. 2010, MNRAS, 404, 1111
Han, J., Jing, Y., Wang, H., \& Wang, W. 2011, in Galaxy Formation: An International Conference, Online at ¡A href="http://astro.dur.ac.uk/Gal2011"';

http://astro.dur.ac.uk/Gal2011 $\mathrm{i}_{\mathrm{i}} \mathrm{A}_{i}$, id.P175, 175P

Hawkins, E., Maddox, S., Cole, S., et al. 2003, MNRAS, 346, 78

Hayashi, E., \& White, S. D. M. 2008, MNRAS, 388, 2

Jing, Y. P., \& Börner, G. 2004, ApJ, 617, 782

Jing, Y. P., Mo, H. J., \& Boerner, G. 1998, ApJ, 494, 1

Jing, Y. P., \& Suto, Y. 1998, ApJ, 494, L5

-. 2000, ApJ, 529, L69

-. 2002, ApJ, 574, 538

Jing, Y. P., Suto, Y., \& Mo, H. J. 2007, ApJ, 657, 664

Kaiser, N. 1987, MNRAS, 227, 1

Kauffmann, G., Heckman, T. M., White, S. D. M., et al. 2003, MNRAS, 341,33

Kochanek, C. S. 1996, ApJ, 457, 228

Komatsu, E., Smith, K. M., Dunkley, J., et al. 2011, ApJS, 192, 18

Koopmans, L. V. E., Treu, T., Bolton, A. S., Burles, S., \& Moustakas, L. A. 2006, ApJ, 649, 599

Lewis, A., Challinor, A., \& Lasenby, A. 2000, ApJ, 538, 473

Li, C., Jing, Y. P., Kauffmann, G., et al. 2007, MNRAS, 376, 984

-. 2006a, MNRAS, 368, 37

Li, C., Kauffmann, G., Fu, J., et al. 2012, MNRAS, 424, 1471

Li, C., Kauffmann, G., Jing, Y. P., et al. 2006b, MNRAS, 368, 21

Li, C., Kauffmann, G., Wang, L., et al. 2006c, MNRAS, 373, 457

Li, C., \& White, S. D. M. 2009, MNRAS, 398, 2177

Lilje, P. B., \& Efstathiou, G. 1989, MNRAS, 236, 851

Lin, W. P., Jing, Y. P., Mao, S., Gao, L., \& McCarthy, I. G. 2006, ApJ, 651, 636

Łokas, E. L., \& Mamon, G. A. 2003, MNRAS, 343, 401

Mandelbaum, R., Seljak, U., Kauffmann, G., Hirata, C. M., \& Brinkmann, J. 2006, MNRAS, 368, 715

Marzke, R. O., Geller, M. J., da Costa, L. N., \& Huchra, J. P. 1995, AJ, 110, 477

Masjedi, M., Hogg, D. W., Cool, R. J., et al. 2006, ApJ, 644, 54

McKay, T. A., Sheldon, E. S., Johnston, D., et al. 2002, ApJ, $571, \mathrm{~L} 85$

Mo, H., van den Bosch, F. C., \& White, S. 2010, Galaxy Formation and Evolution

Mo, H. J., Jing, Y. P., \& Boerner, G. 1992, ApJ, 392, 452

Mo, H. J., Jing, Y. P., \& Borner, G. 1993, MNRAS, 264, 825

More, S., van den Bosch, F. C., \& Cacciato, M. 2009a, MNRAS, 392, 917

More, S., van den Bosch, F. C., Cacciato, M., et al. 2009b, MNRAS, 392, 801

- 2011, MNRAS, 410,210

Moster, B. P., Somerville, R. S., Maulbetsch, C., et al. 2010, ApJ, 710,903

Navarro, J. F., Frenk, C. S., \& White, S. D. M. 1997, ApJ, 490, 493

Neistein, E., Li, C., Khochfar, S., et al. 2011a, MNRAS, 416, 1486

Neistein, E., Weinmann, S. M., Li, C., \& Boylan-Kolchin, M. 2011b, MNRAS, 414, 1405

Norberg, P., Frenk, C. S., \& Cole, S. 2008, MNRAS, 383, 646

Okumura, T., \& Jing, Y. P. 2011, ApJ, 726, 5

Peebles, P. J. E. 1980, The large-scale structure of the universe

Prada, F., Vitvitska, M., Klypin, A., et al. 2003, ApJ, 598, 260

Press, W. H., \& Schechter, P. 1974, ApJ, 187, 425

Samushia, L., Percival, W. J., \& Raccanelli, Á. 2012, MNRAS, 420,2102

Schlegel, D., Abdalla, F., Abraham, T., et al. 2011, e-print at arXiv: 1106.1706 
Shankar, F., Lapi, A., Salucci, P., De Zotti, G., \& Danese, L. 2006, ApJ, 643, 14

Skibba, R. A., van den Bosch, F. C., Yang, X., et al. 2011, MNRAS, 410, 417

Slosar, A., Seljak, U., \& Tasitsiomi, A. 2006, MNRAS, 366, 1455

Somerville, R. S., Davis, M., \& Primack, J. R. 1997, ApJ, 479, 616

Tal, T., Wake, D. A., \& van Dokkum, P. G. 2012, ApJ, 751, L5

Tinker, J. L. 2007, MNRAS, 374, 477

Tinker, J. L., Norberg, P., Weinberg, D. H., \& Warren, M. S. 2007, ApJ, 659, 877

Vale, A., \& Ostriker, J. P. 2004, MNRAS, 353, 189

van den Bosch, F. C., Norberg, P., Mo, H. J., \& Yang, X. 2004, MNRAS, 352, 1302

van den Bosch, F. C., Weinmann, S. M., Yang, X., et al. 2005 MNRAS, 361, 1203

van den Bosch, F. C., Yang, X., Mo, H. J., et al. 2007, MNRAS, 376,841

van der Marel, R. P., Magorrian, J., Carlberg, R. G., Yee, H. K. C., \& Ellingson, E. 2000, AJ, 119, 2038

von der Linden, A., Allen, M. T., Applegate, D. E., et al. 2012, submitted to MNRAS, e-print at arXiv:1208.0597

Wang, J., De Lucia, G., Kitzbichler, M. G., \& White, S. D. M. 2008, MNRAS, 384, 1301

Wang, L., Li, C., Kauffmann, G., \& De Lucia, G. 2006, MNRAS, 371,537

. 2007, MNRAS, 377, 1419

Watson, D. F., Berlind, A. A., McBride, C. K., Hogg, D. W., \& Jiang, T. 2012, ApJ, 749, 83

Watson, D. F., Berlind, A. A., McBride, C. K., \& Masjedi, M. 2010, ApJ, 709, 115
White, S. D. M., \& Rees, M. J. 1978, MNRAS, 183, 341

Wilkinson, M. I., \& Evans, N. W. 1999, MNRAS, 310, 645

Yahil, A. 1985, in European Southern Observatory Conference and Workshop Proceedings, ed. O.-G. Richter \& B. Binggeli, Vol. 20, 359-373

Yang, X., Mo, H. J., Jing, Y. P., van den Bosch, F. C., \& Chu, Y. 2004, MNRAS, 350, 1153

Yang, X., Mo, H. J., \& van den Bosch, F. C. 2009, ApJ, 695, 900

Yang, X., Mo, H. J., van den Bosch, F. C., \& Jing, Y. P. 2005a, MNRAS, 356, 1293

Yang, X., Mo, H. J., van den Bosch, F. C., et al. 2007, ApJ, 671, 153

. 2005b, MNRAS, 362, 711

Yang, X., Mo, H. J., van den Bosch, F. C., Zhang, Y., \& Han, J. 2012, ApJ, 752, 41

York, D. G., Adelman, J., Anderson, Jr., J. E., et al. 2000, AJ, 120,1579

Yoshikawa, K., Jing, Y. P., \& Börner, G. 2003, ApJ, 590, 654

Zaritsky, D., Smith, R., Frenk, C., \& White, S. D. M. 1993, ApJ, 405,464

-. 1997, ApJ, 478, 39

Zaritsky, D., \& White, S. D. M. 1994, ApJ, 435, 599

Zehavi, I., Blanton, M. R., Frieman, J. A., et al. 2002, ApJ, 571 172

Zehavi, I., Zheng, Z., Weinberg, D. H., et al. 2005, ApJ, 630, 1

Zhao, D. H., Jing, Y. P., Mo, H. J., \& Börner, G. 2009, ApJ, 707, 354

Zurek, W. H., Quinn, P. J., Salmon, J. K., \& Warren, M. S. 1994, ApJ, 431, 559 